\title{
Mon-735: a new low-mass pre-main-sequence eclipsing binary in NGC 2264
}

\author{
Edward Gillen ${ }^{\oplus},{ }^{1 \star} \dagger$ Lynne A. Hillenbrand, ${ }^{2}$ John Stauffer ${ }^{\odot}, 3$ Suzanne Aigrain ${ }^{\oplus},{ }^{4}$ \\ Luisa Rebull $^{\oplus 5}$ and Ann Marie Cody ${ }^{\oplus 6}$ \\ ${ }^{1}$ Astrophysics Group, Cavendish Laboratory, J.J. Thomson Avenue, Cambridge CB3 OHE, UK \\ ${ }^{2}$ Department of Astronomy, California Institute of Technology, Pasadena, CA 91125, USA \\ ${ }^{3}$ Spitzer Science Center, California Institute of Technology, 1200 E California Blvd., Pasadena, CA 91125, USA \\ ${ }^{4}$ Sub-department of Astrophysics, Department of Physics, University of Oxford, Keble Road, Oxford OX1 3RH, UK \\ ${ }^{5}$ Infrared Science Archive (IRSA), IPAC, California Institute of Technology, 1200 E California Blvd., Pasadena, CA 91125, USA \\ ${ }^{6}$ NASA Ames Research Center, Moffet Field, CA 94035, USA
}

\begin{abstract}
We present Mon-735, a detached double-lined eclipsing binary (EB) member of the $\sim 3$ Myr old NGC 2264 star-forming region, detected by Spitzer. We simultaneously model the Spitzer light curves, follow-up Keck/HIRES radial velocities, and the system's spectral energy distribution to determine self-consistent masses, radii, and effective temperatures for both stars. We find that Mon-735 comprises two pre-main-sequence $\mathrm{M}$ dwarfs with component masses of $M=$ $0.2918 \pm 0.0099$ and $0.2661 \pm 0.0095 \mathrm{M}_{\odot}$, radii of $R=0.762 \pm 0.022$ and $0.748 \pm 0.023 \mathrm{R}_{\odot}$, and effective temperatures of $T_{\text {eff }}=3260 \pm 73$ and $3213 \pm 73 \mathrm{~K}$. The two stars travel on circular orbits around their common centre of mass in $P=1.9751388 \pm 0.0000050 \mathrm{~d}$. We compare our results for Mon-735, along with another EB in NGC 2264 (CoRoT 223992193), to the predictions of five stellar evolution models. These suggest that the lower mass EB system Mon-735 is older than CoRoT 223992193 in the mass-radius diagram (MRD) and, to a lesser extent, in the Hertzsprung-Russell diagram (HRD). The MRD ages of Mon-735 and CoRoT 223992193 are 7-9 and 4-6 Myr, respectively, with the two components in each EB system possessing consistent ages.
\end{abstract}

Key words: binaries: eclipsing - binaries: spectroscopic-stars: fundamental parameters stars: low-mass-stars: pre-main-sequence-open clusters and associations: individual: NGC 2264.

\section{INTRODUCTION}

Accurately predicting the fundamental properties of stars (e.g. masses, radii, effective temperatures, luminosities, and ages) is a central requirement for observational astrophysics. Modern stellar evolution theory, for example, underpins our ability to accurately estimate: the initial mass function (e.g. Bastian, Covey \& Meyer 2010); determine cluster and association ages (e.g. Soderblom et al. 2014; Bell, Mamajek \& Naylor 2015) and hence protoplanetary disc lifetimes and giant planet formation time-scales (e.g. Alexander 2008; Williams \& Cieza 2011; Ribas, Bouy \& Merín 2015); infer exoplanet parameters (e.g. Gaidos \& Mann 2013; Jones et al. 2016; Raynard et al. 2018; Rickman et al. 2019); and constrain the age-

\footnotetext{
^E-mail: ecg41@cam.ac.uk

$\dagger$ Winton Fellow.
}

activity-rotation relation (e.g. Barnes et al. 2005; Meibom, Mathieu \& Stassun 2009; Meibom et al. 2015). Uncertainties in modelderived stellar properties translate into systematic uncertainties in these areas. Significant effort is therefore directed into observationally testing stellar evolution models, especially at low masses $\left(M<0.8 \mathrm{M}_{\odot}\right)$ and young ages $(t<1 \mathrm{Gyr})$, as current constraints remain scarce.

Detached, double-lined eclipsing binary (EB) stars offer a useful test of stellar models, as the masses and radii of both stars can be directly measured, with minimal theoretical assumptions, from the light and radial velocity (RV) curves of the system. With high-quality data and modern software tools, fundamental stellar parameters can be measured to precisions of 1-3 per cent; these provide one of the strongest observational tests on stellar evolution theory available (Andersen 1991; Torres, Andersen \& Giménez 2010; Stassun, Feiden \& Torres 2014). Furthermore, with light curves in two or more (ideally well-separated) photometric bands, 
effective temperature ( $T_{\text {eff }}$ ) ratios can be determined from relative eclipse depths, albeit it with some dependence on either stellar atmosphere models or empirical relations (and to obtain absolute $T_{\text {eff }} \mathrm{S}$ would require one component to be determined independently). Nonetheless, by simultaneously analysing photometric eclipses (ideally in multiple bands), RVs, and either spectral energy distributions (SEDs) or flux-calibrated spectra (or by performing spectral disentangling), it is possible to self-consistently solve for the stellar masses, radii, effective temperatures, and luminosities. Finally, parallax measurements (e.g. from Gaia; Gaia Collaboration et al. 2018) give independent distance estimates that can be incorporated into the fit.

Open clusters are stellar populations that share the same age and metallicity, and thereby represent powerful tests of stellar evolution theory due to their ensemble nature. Young open clusters offer a window on to the early evolution of stellar and planetary systems and, as their member stars can be dated to a precision unattainable for young stars in the field, constrain time-scales for the processes driving their evolution. Consequently, many photometric monitoring programs have targeted young open clusters to: study accretion and the star-disc interaction (e.g. Bouvier et al. 2007; Alencar et al. 2010; Cody et al. 2014; Stauffer et al. 2014, 2015); understand the evolution of stellar rotation (e.g. Irwin et al. 2006; Meibom et al. 2009; Hartman et al. 2010; Affer et al. 2013; Rebull et al. 2016a,b; Stauffer et al. 2016; Douglas et al. 2017; Gillen et al. 2020) and pulsations (e.g. Zwintz \& Weiss 2006; Zwintz et al. 2011); and to search for young transiting planets (e.g. David et al. 2016b; Mann et al. 2017; Pepper et al. 2017; Livingston et al. 2018), brown dwarfs (e.g. Gillen et al. 2017b; David et al. 2019), and EBs (e.g. Stassun et al. 2004; Irwin et al. 2007; Gillen et al. 2014; Kraus et al. 2015, 2017; David et al. 2016a).

EBs in open clusters are particularly valuable because their metallicity can be inferred through their membership, and their derived age acts as an independent age estimate for the cluster. Multiple EBs in the same cluster, which share the same cluster age and metallicity, are even more valuable as they can act as a joint test on stellar evolution theory (e.g. Gillen et al. 2017b; David et al. 2019). The search for new EBs in young open clusters was one of the primary science goals behind the Convection, Rotation and planetary Transits (CoRoT) space mission (Baglin 2003) observing the NGC 2264 star-forming region for $23 \mathrm{~d}$ in 2008, as well as subsequent simultaneous CoRoT and Spitzer (Werner et al. 2004) observations of NGC 2264 in 2011-2012 (39 and 29 d, respectively). These observations detected several tens of EBs, most of which are field-age systems, with Gillen et al. (2014) presenting the first confirmed low-mass EB member of the cluster. Here, we focus on the second low-mass EB member of NGC 2264, Mon-735, which was detected through the 2011-2012 Spitzer observations. The position of Mon-735 was not covered by the aforementioned CoRoT observations.

The NGC 2264 star-forming region is the dominant component of the Mon OB1 association in the Monoceros constellation, which is situated in the local Orion-Cygnus spiral arm at a distance of $\sim 700$ 800 pc (Sung, Bessell \& Lee 1997; Dahm 2008; Sung \& Bessell 2010; Gillen et al. 2014). NGC 2264 is hierarchically structured with significant subclusterings and a halo population (e.g. Teixeira et al. 2006). It comprises $\sim 1500$ stars and has a commonly accepted median age of $\sim 3 \mathrm{Myr}$ with an apparent dispersion of $\sim 3-5 \mathrm{Myr}$ (Walker 1956; Park et al. 2000; Rebull et al. 2002). This age dispersion arises from the fact that early-type members (A0 and earlier) are believed to have reached the main sequence, which sets a minimum cluster age, and yet star formation is ongoing (Young et al. 2006;
Table 1. Names and coordinates for Mon-735.

\begin{tabular}{lll}
\hline Property & \multicolumn{1}{c}{ Value } & Epoch \\
\hline Names & CSI Mon-000735 & \\
& 2MASS J06412734+0942003 & \\
& Gaia DR2 3326699526510395264 & J2000.0 \\
RA & 06 41 27.342 & J2000.0 \\
Dec. & +094200.378 & \\
\hline
\end{tabular}

Dahm 2008; Sung \& Bessell 2010). NGC 2264 has been historically well studied due to its large and well-defined membership, and lowforeground extinction of $E(B-V)=0.06-0.15$ mag (e.g. Perez, The \& Westerlund 1987; Rebull et al. 2002; Mayne \& Naylor 2008). The cluster RV is formally estimated as $\mathrm{RV}=22 \pm 3.5 \mathrm{~km} \mathrm{~s}^{-1}$, but displays a significantly non-Gaussian dispersion where stars in the range $\mathrm{RV}=8-36 \mathrm{~km} \mathrm{~s}^{-1}$ have been considered as potential members (Fürész et al. 2006; Tobin et al. 2015).

This paper presents the discovery and characterization of Mon735 as a young low-mass pre-main-sequence (PMS) EB member of NGC 2264. The source has been previously identified as a T Tauri-type member of NGC 2264 based on ultraviolet excess and/or optical variability (Rebull et al. 2002; Lamm et al. 2004; Barentsen et al. 2013; Venuti et al. 2014) and is listed as an EB in the surrounding CoRoT SRa01 field (Klagyivik et al. 2013). In Section 2, we introduce the photometric and spectroscopic observations. In Section 3, we model the light curves, RVs, and SED of the system to determine masses, radii, temperatures, and luminosities for both stars. We report our results in Section 4 along with a reanalysis of CoRoT 223992193 to obtain a self-consistent set of parameters for both systems. In Section 5, we compare our fundamental parameters for both stars with the predictions of five modern stellar evolution models before concluding in Section 6.

\section{OBSERVATIONS}

The NGC 2264 star-forming region was the subject of a photometric and spectroscopic monitoring campaign from 15 ground- and spacebased telescope facilities in late 2011. This Coordinated Synoptic Investigation of NGC 2264 (CSI 2264) is introduced in detail in Cody et al. (2014). Here, we focus on the Spitzer/Infrared Array Camera (IRAC) 3.6 and $4.5 \mu \mathrm{m}$ observations (PI: Stauffer), which discovered the Mon-735 EB system, and follow-up spectroscopic measurements with the Keck/High Resolution Echelle Spectrometer (HIRES) spectrograph. The names, coordinates, and broad-band magnitudes of Mon-735 are given in Tables 1 and 2.

\subsection{Photometry}

Mon-735 was observed by Spitzer/IRAC at 3.6 and $4.5 \mu \mathrm{m}$ for 28.7 d between 4 December 2011 and 1 January 2012 at a typical cadence of $1.7 \mathrm{~h}$. The 3.6 and $4.5 \mu \mathrm{m}$ observations are nearsimultaneous, with the $4.5 \mu \mathrm{m}$ data taken $\sim 20 \mathrm{~min}$ after the $3.6 \mu \mathrm{m}$ observations. Spitzer performed four 20-h continuous (stare mode) observations towards the beginning of the campaign, leading to corresponding data gaps in the photometry of Mon-735, as it did not fall within the continuous observation region. The Spitzer data were converted from magnitudes into relative flux units for the analysis presented here. The reader is referred to Cody et al. (2014) 
Table 2. Broad-band system photometry of Mon-735.

\begin{tabular}{|c|c|c|c|}
\hline Band & Magnitude & $\begin{array}{l}\text { Spectral flux density } \\
\left(\mathrm{erg} \mathrm{s}^{-1} \mathrm{~cm}^{-2} \AA^{-1}\right)\end{array}$ & Refs. \\
\hline SDSS $u$ & $22.018 \pm 0.201 \mathrm{AB}$ & $(1.35 \pm 0.25) \times 10^{-17}$ & $(1,5,6)$ \\
\hline SDSS $g$ & $19.850 \pm 0.016 \mathrm{AB}$ & $(5.732 \pm 0.084) \times 10^{-17}$ & $(1,5,6)$ \\
\hline SDSS $r$ & $18.446 \pm 0.009 \mathrm{AB}$ & $(1.202 \pm 0.010) \times 10^{-16}$ & $(1,5,6)$ \\
\hline SDSS $i$ & $16.892 \pm 0.005 \mathrm{AB}$ & $(3.415 \pm 0.016) \times 10^{-16}$ & $(1,5,6)$ \\
\hline $\operatorname{SDSS} z$ & $16.040 \pm 0.007 \mathrm{AB}$ & $(5.252 \pm 0.034) \times 10^{-16}$ & $(1,5,6)$ \\
\hline Pan-STARRS1 $g$ & $19.700 \pm 0.037 \mathrm{AB}$ & $(6.10 \pm 0.21) \times 10^{-17}$ & $(2,5,7)$ \\
\hline Pan-STARRS $1 r$ & $18.449 \pm 0.010 \mathrm{AB}$ & $(1.181 \pm 0.011) \times 10^{-16}$ & $(2,5,7)$ \\
\hline Pan-STARRS1 $i$ & $16.882 \pm 0.005 \mathrm{AB}$ & $(3.388 \pm 0.014) \times 10^{-16}$ & $(2,5,7)$ \\
\hline Pan-STARRS $1 z$ & $16.177 \pm 0.002 \mathrm{AB}$ & $(4.895 \pm 0.010) \times 10^{-16}$ & $(2,5,7)$ \\
\hline Pan-STARRS $1 y$ & $15.829 \pm 0.009 \mathrm{AB}$ & $(5.475 \pm 0.047) \times 10^{-16}$ & $(2,5,7)$ \\
\hline Gaia $G$ & $17.6210 \pm 0.0025$ & $(2.2763 \pm 0.0052) \times 10^{-16}$ & $(3,5,8)$ \\
\hline Gaia $G_{\mathrm{BP}}$ & $19.0371 \pm 0.0444$ & $(1.008 \pm 0.041) \times 10^{-16}$ & $(3,5,8)$ \\
\hline Gaia $G_{\mathrm{RP}}$ & $16.3417 \pm 0.0083$ & $(3.825 \pm 0.029) \times 10^{-16}$ & $(3,5,8)$ \\
\hline 2MASS $J$ & $14.491 \pm 0.029$ & $(5.00 \pm 0.13) \times 10^{-16}$ & $(4,5,9)$ \\
\hline 2MASS $H$ & $13.910 \pm 0.025$ & $(3.093 \pm 0.071) \times 10^{-16}$ & $(4,5,9)$ \\
\hline 2MASS $K_{\mathrm{S}}$ & $13.520 \pm 0.039$ & $(1.674 \pm 0.060) \times 10^{-16}$ & $(4,5,9)$ \\
\hline Spitzer IRAC-1 & $13.424 \pm 0.015$ & $(2.873 \pm 0.040) \times 10^{-17}$ & (This work, 5, 10) \\
\hline Spitzer IRAC-2 & $13.342 \pm 0.015$ & $(1.237 \pm 0.017) \times 10^{-17}$ & (This work, 5,10 ) \\
\hline \multicolumn{4}{|c|}{$\begin{array}{l}\text { Note. Magnitudes and spectral flux densities typically have formal measurement errors, which do not reflect } \\
\text { the system's intrinsic variability. The Sloan Digital Sky Survey (SDSS) and Panoramic Survey Telescope } \\
\text { and Rapid Response System (Pan-STARRS) magnitudes are in the AB system, while Gaia, Two Micron } \\
\text { All-Sky Survey (2MASS), and Spitzer magnitudes are in Vega. } \\
\text { References. Photometry: } 1 \text { - Aguado et al. (2019); } 2 \text { - Chambers et al. (2016); } 3 \text { - Gaia Collaboration } \\
\text { et al. (2018); } 4 \text { - Skrutskie et al. (2006). Bandpasses: } 5 \text { - Filter Profile Service (FPS; http://svo2.cab.inta- } \\
\text { csic.es/theory/fps); } 6 \text { - SDSS Data Release } 7 \text { (DR7; https://classic.sdss.org/dr7/instruments/imager/\#filte } \\
\text { rs); } 7 \text { - Tonry et al. (2012); } 8 \text { - Evans et al. (2018); } 9 \text { - Cohen, Wheaton \& Megeath (2003); 10 - NASA/IPAC } \\
\text { Infrared Science Archive (https://irsa.ipac.caltech.edu/data/SPITZER/docs/irac). }\end{array}$} \\
\hline
\end{tabular}

Table 3. Radial velocities and flux ratios derived from Keck/HIRES spectra.

\begin{tabular}{lccccrr}
\hline UT date & HJD & Phase $^{a}$ & $\begin{array}{c}\text { S/N } \\
(7550 \AA)\end{array}$ & $\begin{array}{c}\text { Light ratio } \\
(7850-8950 \AA)\end{array}$ & $\begin{array}{r}\text { Primary RV } \\
\left(\mathrm{km} \mathrm{s}^{-1}\right)\end{array}$ & $\begin{array}{r}\text { Secondary RV } \\
\left(\mathrm{km} \mathrm{s}^{-1}\right)\end{array}$ \\
\hline $2015-10-27$ & 2457323.0845 & 0.81 & 18 & $0.895 \pm 0.101$ & $78.19 \pm 3.64$ & $-48.04 \pm 1.53$ \\
$2015-12-24$ & 2457381.0684 & 0.17 & 21 & $0.922 \pm 0.123$ & $-40.48 \pm 3.11$ & $80.98 \pm 2.84$ \\
$2015-12-29$ & 2457385.9827 & 0.65 & 25 & $0.896 \pm 0.122$ & $73.19 \pm 1.46$ & $-44.27 \pm 2.99$ \\
$2016-02-02$ & 2457420.8102 & 0.28 & 16 & $0.879 \pm 0.068$ & $-46.39 \pm 1.98$ & $91.45 \pm 2.53$ \\
$2016-02-03$ & 2457421.7907 & 0.78 & 11 & $1.079 \pm 0.058$ & $84.28 \pm 2.98$ & $-52.03 \pm 3.28$ \\
$2016-10-14$ & 2457676.0175 & 0.48 & 13 & - & \multicolumn{2}{c}{$18.58 \pm 1.04$} \\
$2016-12-22$ & 2457744.9145 & 0.38 & 21 & $0.968 \pm 0.131$ & $-28.47 \pm 2.16$ & $69.10 \pm 1.58$ \\
$2017-01-13$ & 2457766.8100 & 0.45 & 17 & $0.865 \pm 0.132$ & $3.64 \pm 1.93$ & $35.32 \pm 1.92$ \\
\hline
\end{tabular}

${ }^{a}$ Phase is defined relative to primary eclipse.

for further details of the Spitzer observations, data reduction, and light-curve production. ${ }^{1}$

\subsection{Spectroscopy}

We obtained eight high-resolution optical spectra with the Keck/HIRES spectrograph (Vogt et al. 1994) between 27 October 2015 and 13 January 2017. The HIRES spectra cover the wavelength range $\sim 4800-9200 \AA$ with a resolving power of $R>36000$, and were reduced using the dedicated MAKEE software written by Tom Barlow.

Details of the individual observations are given in Table 3 along with information from the analysis presented in Section 3.2. The Mon-735 system is double lined in the HIRES spectra at all epochs

\footnotetext{
${ }^{1}$ See https://irsa.ipac.caltech.edu/data/SPITZER/CSI2264/ for the Spitzer
} data archive. bar one (2016-10-14), where only a single peak was apparent, corresponding to both components of the system being located close to the systematic velocity. We determined a spectral type of M3.1 \pm 0.7 for this composite spectrum by measuring the strength of several $\mathrm{TiO}$ features relative to a set of spectral standards. The analytically determined spectral type was verified by visual examination of the spectrum details over a range of wavelengths.

The spectra display weak $\mathrm{H} \alpha$ and $\mathrm{H} \beta$ emission, as well as LiI $6708 \AA$ absorption, which together suggest Mon-735 is a chromospherically active young system (Stauffer \& Hartmann 1986; West et al. 2011; Soderblom et al. 2014). Examples of these three spectral features at different phases of the EB orbit are shown in Fig. 1. The H $\alpha$ equivalent width is about $-2.5 \AA$ for each component of the EB, with line profiles that are flat-topped, as is often seen in chromospherically active moderately rotating mid-M-type stars (see examples in appendix A of López-Santiago et al. 2010). The Ca II triplet lines are purely in absorption with no 

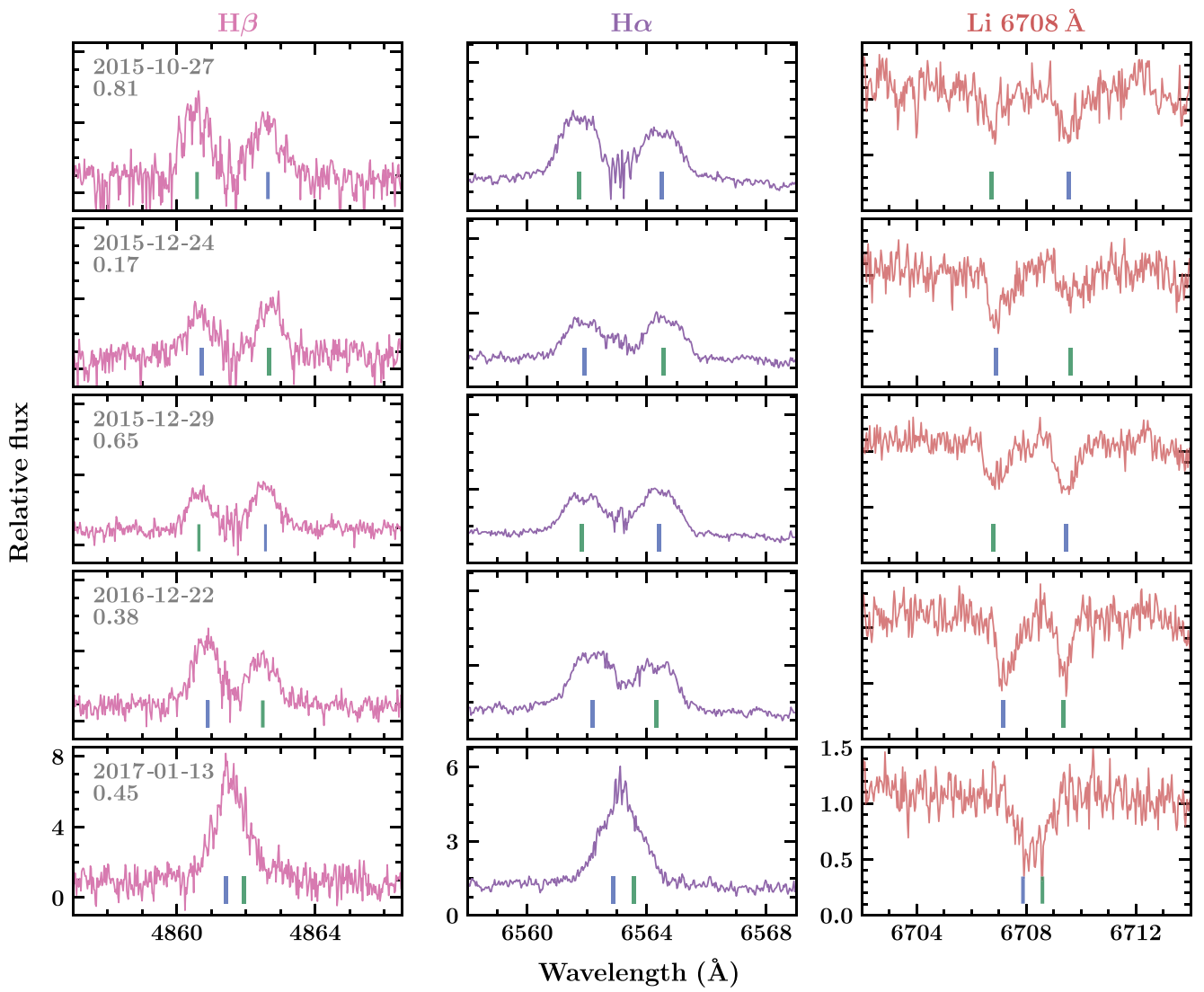

Figure 1. $\mathrm{H} \beta, \mathrm{H} \alpha$, and Li I $6708 \AA ̊ ̊$ profiles of Mon-735 (columns, left-to-right) for different epochs (rows, top-to-bottom). The epoch and orbital phase are given in the $\mathrm{H} \beta$ plots. The vertical blue and green lines indicate the radial velocities (RVs) of the primary and secondary stars, respectively, at each epoch. Both stars present $\mathrm{H} \beta$ and $\mathrm{H} \alpha$ emission profiles, and Li I $6708 \AA$ absorption profiles, which suggest that Mon-735 is a chromospherically active young system.

obvious central line reversal; thus neither component of the system seems to be highly active (Schöfer et al. 2019), or an accretor (Herbig \& Soderblom 1980). The Li I $6708 \AA$ absorption strength is approximately 290 and $320 \mathrm{~mA}$ in the two components, which is typical for the pseudo-equivalent width of NGC 2264 members at this spectral type, however, somewhat low for truly measured equivalent widths that account for the significant TiO absorption in this spectral region (Bouvier et al. 2016).

\section{ANALYSIS WITH GP - EBOP}

GP-EBOP is an EB and transiting planet model that is optimized for modelling young and/or active systems. Its main motivation is to account for the effect of stellar activity in the determination of stellar or planetary fundamental parameters. It is introduced in detail in Gillen et al. (2017b, hereafter G17) and we refer the interested reader there. Here, we briefly recap the main points of the model and highlight some new improvements implemented since G17.

GP-EBOP comprises a Gaussian process (GP) framework, which contains an EB and transiting planet model, and has a Markov chain Monte Carlo (MCMC) wrapper. The central EB model uses EB (Irwin et al. 2011, 2018), which is a descendent of the EBOP family of models, but which uses the analytic method of Mandel \& Agol (2002) to perform the eclipse calculations. GP-EBOP further parametrizes the eccentricity and longitude of periastron terms using their combinations $\sqrt{e} \cos \omega$ and $\sqrt{e} \sin \omega$, rather than $e \cos \omega$ and $e \sin \omega$, to avoid inducing a prior on the orbital eccentricity proportional to $e$ (Ford 2006; Triaud et al. 2011). Limb darkening is typically parametrized using the triangular sampling method of Kipping (2013), with theoretical constraints applied based on the predictions of the Limb Darkening Toolkit (LDTK; Parviainen \& Aigrain 2015) for optical and near-infrared (IR) bandpasses. The MCMC model uses the affine invariant method implemented in EMCEE (Foreman-Mackey et al. 2013).

There are two main updates to GP-EBOP since G17.

(i) The GP model is now updated to use the CELERITE library (Foreman-Mackey et al. 2017), which allows fast and scalable GP regression in one dimension.

(ii) GP-EBOP now has the capability to simultaneously model SEDs along with light curves and RVs.

For the present analysis, we simultaneously modelled the observed light curves, RVs, and SED of Mon-735. Jointly modelling the SED allows us to propagate our optical spectroscopic light ratio constraints into the IR Spitzer light-curve bands and use them to help constrain the fundamental parameters of both stars. This is particularly important for Mon-735, as the system displays nearequal eclipse depths in the Spitzer light curves, which can lead to a degeneracy between the radius ratio, surface brightness ratio, and inclination when modelling the data. Details of the modelling are given in Sections 3.1-3.3 below.

\subsection{Light curves}

The Spitzer 3.6 and $4.5 \mu \mathrm{m}$ light curves are shown in Fig. 2 . In addition to eclipses on both the primary and secondary stars, 

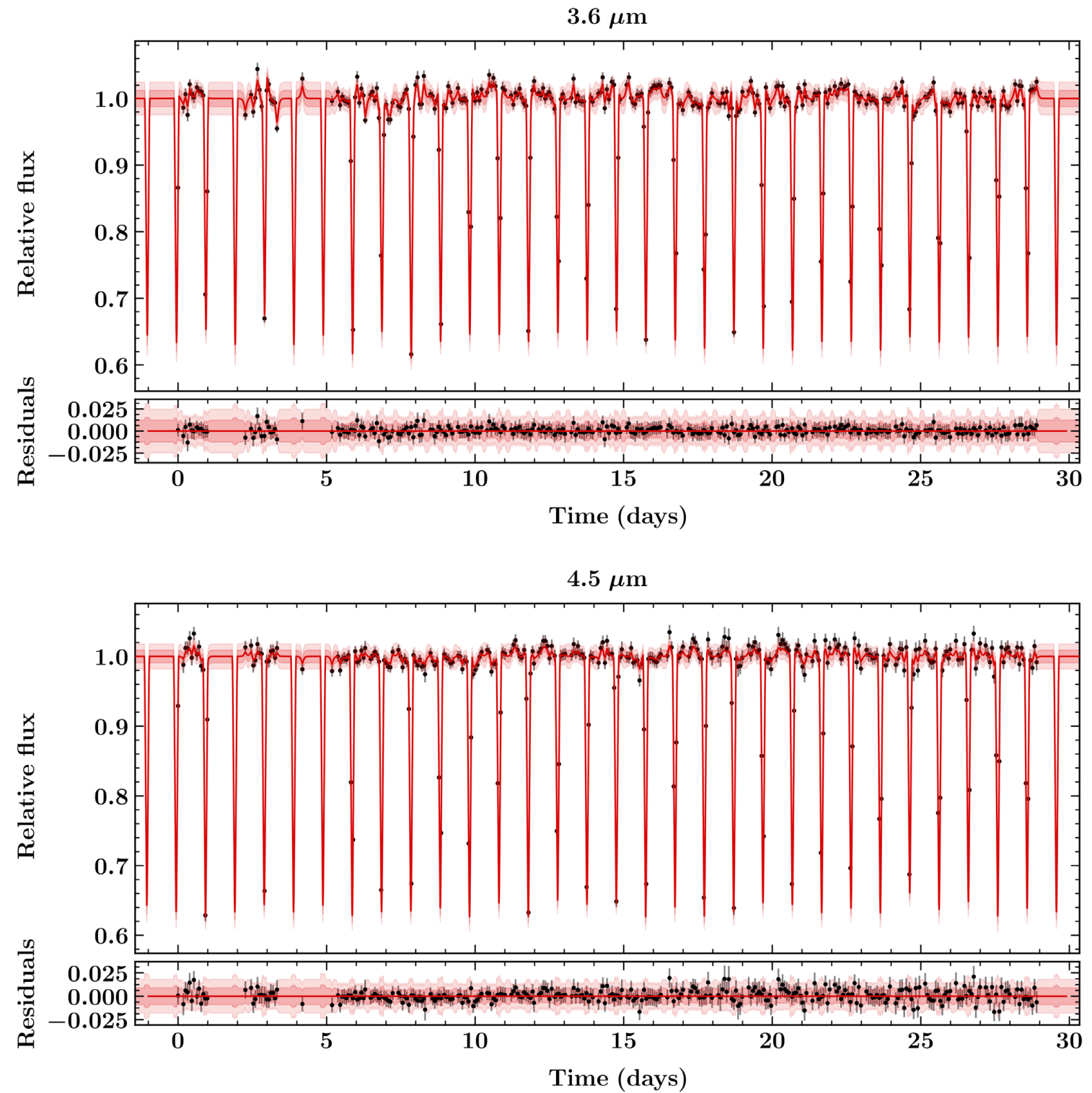

Figure 2. Spitzer/IRAC 3.6 and $4.5 \mu \mathrm{m}$ relative flux light curves of Mon-735 (black points, top and bottom, respectively) with the GP-EBOP model in red and residuals below. In both light curves, the red line and pink shaded regions show the mean and $1 \sigma$ and $2 \sigma$ confidence intervals of the predictive posterior distribution. The system displays near-equal eclipses on both stars, which are $\sim 36-37$ per cent deep, as well as relatively rough short time-scale out-of-eclipse variability.

both light curves display reasonably rough aperiodic out-of-eclipse (OOE) variations, which are similar to Spitzer light curves of other young EBs (e.g. Morales-Calderón et al. 2012; Gillen et al. 2017a). Given the rough OOE variations, we opted to use a Matérn kernel with smoothness parameter $v=3 / 2$ (as approximated within the CELERITE package), which is appropriate for such rough variations (Rasmussen \& Williams 2006). The Matérn 3/2 covariance kernel $k$ is given by

$k_{\mathrm{M} 32}(\tau)=A^{2}\left(1+\frac{\sqrt{3} \tau}{l}\right) \exp \left(-\frac{\sqrt{3} \tau}{l}\right)$,

where $\tau=\left|t_{i}-t_{j}\right|$ is the time interval between times $t_{i}$ and $t_{j}$, and $A$ and $l$ are the characteristic amplitude and time-scale of the variations, respectively.

We applied theoretical constraints on the quadratic limb darkening parameters using the predictions of Claret, Hauschildt \& Witte
(2012). We used the Claret tables rather than LDTK because the specific intensities of the PHOENIX model spectra (Husser et al. 2013), which LDTK is based on, only extend up to a wavelength of $2.6 \mu \mathrm{m}$, so the IRAC 3.6 and $4.5 \mu \mathrm{m}$ bandpasses are not covered. We assumed $T_{\text {eff }}=3200 \mathrm{~K}$ and $\log g=4.0$ for both the primary and secondary stars (setting $Z=0.0$, which is the closest value available to the cluster metallicity; King et al. 2000). ${ }^{2}$ The $T_{\text {eff }}$ and $\log g$ values were set based on preliminary fits to the SED data (using PHOENIX models) and the light curves and RVs, respectively, and then rounded to the nearest value in the Claret tables. Uncertainties on the limb darkening coefficients (LDCs) were set based on the spread of coefficient values within a representative range in $T_{\text {eff }}$ and

${ }^{2} \mathrm{NGC} 2264$ is suggested to have $[\mathrm{Fe} / \mathrm{H}]=-0.15$ with near-solar ratios of other elements. 
$\log g$, which were determined from initial modelling of the light curves and RVs, and further inflated by comparing the Claret LDC values using both the least-square and flux conservation methods. In practice, given the IR bandpasses and grazing eclipses, the exact choice of LDCs does not significantly affect the fundamental stellar parameters.

\subsection{Radial velocities}

For each Keck/HIRES spectrum, spectral orders between 6200 and $7200 \AA$ A were used to determine RVs for both components of Mon735 via cross-correlation. In each analysed order, the spectral range was restricted to avoid the edges of orders and telluric lines. The task FXCOR within IRAF was used to measure relative velocities between Mon-735 and a set of $\sim \mathrm{M} 2-\mathrm{M} 4 \mathrm{RV}$ reference stars (i.e. well matched to our M3 source). The number of reference stars ranged between 2 and 14, depending on the observation date, with GJ 105B, GJ 388, GJ 176, and GJ 411 the most typically sampled stars over the many nights and runs. Correlation peaks were measured using either Gaussian or parabolic fits, depending on the signal-to-noise ratio $(\mathrm{S} / \mathrm{N})$. The resulting $\mathrm{RV}$ time series was modelled with Keplerian orbits.

The correlation peaks were further used to establish the red optical flux ratio at the measurement epochs. We used the ratio of the two cross-correlation peak heights to give an estimate of the light ratio between the two stars in the 7850-8950 range. Spectral disentangling is an alternative approach to estimate stellar parameters for each star individually and hence is amenable to estimating flux ratios. It has been successfully applied to more massive systems (see e.g. Pavlovski, Southworth \& Tamajo 2018; Johnston, Pavlovski \& Tkachenko 2019), as well as low-mass objects through the use of GPs (Czekala et al. 2017), and we therefore see it as an interesting avenue for further work.

Spectroscopic light ratios can be used as a prior during the light curve and RV fitting process. They are particularly useful for EBs with near-equal eclipse depths, as they can help break the degeneracy between the radius and surface brightness ratios, and also the inclination. Typically, such spectroscopic light ratios are used as direct priors on the light-curve solution, under the assumption that the spectroscopic wavelength range is an acceptable proxy for the observed light-curve bandpass. Here, however, this is not possible because we have optical spectra and IR light curves. We therefore need a way of propagating the light ratio constraint from the optical spectra into the Spitzer light-curve bands; we do this by simultaneously modelling the SED of the system (i.e. the joint SED of both stars).

\subsection{Spectral energy distribution}

Publicly available broad-band photometry (as reported in Table 2) typically covers the near-ultraviolet (UV) to the near-/mid-IR. This wavelength range covers the rise, peak, and tail of photospheric emission from low-mass stars, which means that SEDs can provide useful constraints on their temperatures. Furthermore, with Gaia Data Release 2 (DR2) parallaxes, SEDs offer complementary information on the stellar radii.

We model the observed SED of Mon-735 as the sum of two stellar photospheres (primary and secondary stars), using BT-Settl model atmospheres (Allard, Homeier \& Freytag 2012) and interpolating the model grid in $T_{\text {eff }}-\log g$ space. ${ }^{3}$ Magnitudes and uncertainties (in either the $\mathrm{AB}$ or Vega systems) were converted to fluxes using the standard relation between magnitude and flux, with their zero-point values and effective wavelengths (defined as the pivot wavelength) obtained from the relevant references in Table 2.

The parameters of the fit were the temperatures, radii and surface gravities of both stars, the distance and reddening to the system, and a jitter term per photometric data set [i.e. Sloan Digital Sky Survey (SDSS), Gaia, Two Micron All-Sky Survey (2MASS)]. The reddening model follows the extinction law of Fitzpatrick (1999), which has been improved by Indebetouw et al. (2005) in the IR [as available through the Filter Profile Service (FPS)]. The SED model used here is essentially an updated version of the model presented in G17, which can now:

(i) accept Gaia parallax measurements to provide distance constraints;

(ii) apply a jitter term per photometric data set (rather than one for the whole SED).

By simultaneously modelling the SED, we can propagate the light ratio constraint from the optical spectra into the Spitzer lightcurve bands. We do this by applying the spectroscopic light ratio as a prior constraint on the atmospheric model light ratio between 7850 and $8950 \AA$, and using the corresponding model flux ratio in the Spitzer bands as the central surface brightness ratio for the eclipse modelling. For the spectroscopic light ratio, we use $l_{\mathrm{sec}} / l_{\mathrm{pri}}$ $=0.896 \pm 0.077$, which is the median and $2 \sigma$ median absolute deviation (MAD)-estimated standard error on the individual values given in Table 3. In addition to enabling the use of a spectroscopic light ratio, modelling the SED allows us to solve for the stellar masses, radii, and temperatures in a self-consistent manner, and to optionally take advantage of the Gaia parallax information when fitting the data.

By including stellar atmosphere models, we make the fitting process slightly model dependent in the sense that we are relying on the stellar atmosphere models to accurately predict flux ratios between the two stars in different photometric bands. This is only a small dependence, however, because while atmosphere models may struggle to reproduce all lines and molecular features, they should be reasonably reliable for predicting the relative flux ratio between two model atmospheres in wide-field photometric bands, such as both the broad-band SED photometry and the light-curve bands are. We note that in Section 4.1, we show for another system (which does not significantly suffer from the aforementioned degeneracies between the radius ratio, inclination, and surface brightness ratio) that modelling either the light curves and RVs only, or also modelling the SED, gives consistent masses and radii for both stars.

Finally, we note that by using stellar atmosphere models with a single temperature for each star, we are not fully accounting for the effect of starspots. Specifically, we are assuming that the overall effect of magnetically active regions to reduce the inferred $T_{\text {eff }}$ (i.e. a combination of filling factor and average spot contrast) is essentially the same on each star, so that the $T_{\text {eff }}$ ratio between the model atmospheres is an acceptable approximation of the true $T_{\text {eff }}$ ratio. For Mon-735, where the two stars are the same age and possess similar masses and radii, it is reasonable to assume their activity

${ }^{3}$ We also tested using the PHOENIX v2 model atmospheres (Husser et al. 2013) but these provided a slightly worse, although still acceptable, fit. Comparing the effect of using BT-Settl or PHOENIX models is discussed in Section 5.5. 

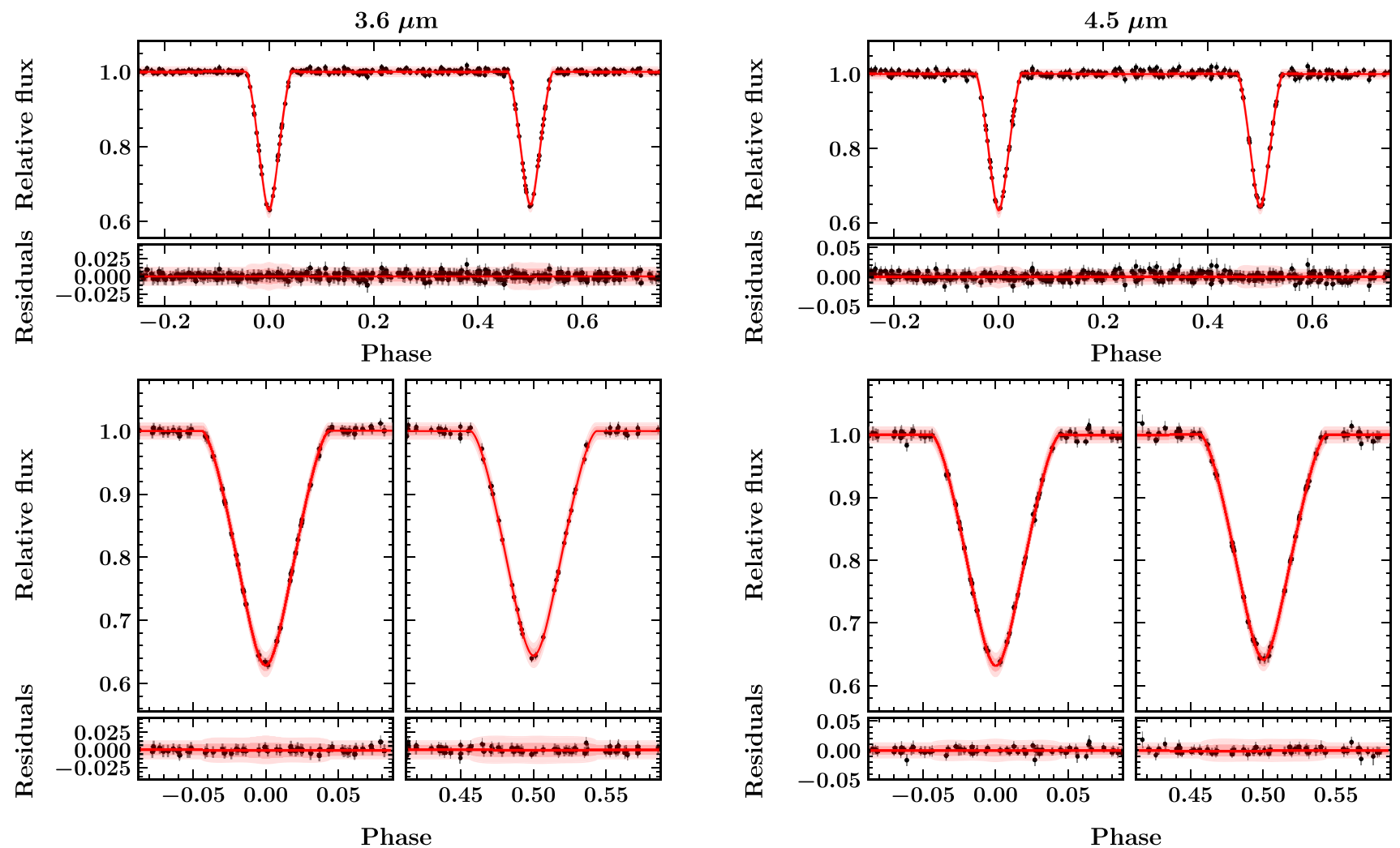

Figure 3. Phase-folded Spitzer/IRAC 3.6 and $4.5 \mu \mathrm{m}$ light curves (left and right, respectively). Top panels: full phase-folded light curves that have been detrended with respect to the Gaussian process model, with residuals immediately below. The red line represents the median posterior EB model, i.e. the median path of individual draws computed in phase space, and the pink shaded regions indicate the $1 \sigma$ and $2 \sigma$ confidence intervals. The phase is defined relative to primary eclipse centre. Bottom panels: zooms on the primary and secondary eclipses (left and right, respectively, for each light curve), with residuals immediately below. The eclipses are grazing with near-equal depths of $\sim 36-37$ per cent on both stars.

levels and hence spot coverage will also be similar. Indeed, the two stars display similar strength line profiles for activity-sensitive lines in the HIRES spectra (see e.g. Fig. 1).

\section{RESULTS}

We simultaneously modelled the Spitzer 3.6 and $4.5 \mu \mathrm{m}$ light curves, Keck/HIRES RVs, and the system SED with GP-EBOP. We used 200 'walkers' to step through and explore the parameter space 50000 times, conservatively discarding the first 30000 steps as 'burn-in', and subsequently thinning each chain following inspection of the autocorrelation lengths for each parameter.

The model fits to the data are shown in Figs 2-5. Fig. 2 shows the Spitzer 3.6 and $4.5 \mu \mathrm{m}$ light curves with the global GP-EBOP model, which achieves an acceptable fit to the rough out-of-eclipse variability in both bands, as well as the eclipses. Fig. 3 shows the detailed fit to the eclipses, which have been phase-folded and detrended with respect to the GP-EBOP variability model. The eclipses are grazing and near-equal in depth at $\sim 36-37$ per cent for both stars. The secondary-to-primary eclipse depth ratio is slightly higher in the $4.5 \mu \mathrm{m}$ band than in $3.6 \mu \mathrm{m}$, which suggests that the secondary star is slightly cooler than the primary. The RV orbit solution is displayed in Fig. 4, phase-folded on the orbital period. The orbits are close to sinusoidal, suggesting a low eccentricity, and the primary RV semi-amplitude is smaller than the secondary's, indicating a higher primary star mass. The RVs are well-fit by Keplerian orbits with residuals of under a few $\mathrm{km} \mathrm{s}^{-1}$. The system SED is shown in Fig. 5 which, peaking at $\sim 1-2 \mu \mathrm{m}$ and appearing similar in shape to that of a single star, suggests that both stars are cool and of similar temperature. The model geometry of the system at primary and secondary eclipse is shown in Fig. 6, highlighting that the two stars have similar radii and that the fraction of each star eclipsed is significant. This configuration is required to explain the deep eclipses on both stars. The main parameters of the fit are given in the top section of Table 4, with derived parameters in the middle and bottom sections.

We find that Mon-735 is composed of two stars with $M_{\text {pri }}=$ $0.2918 \pm 0.0099 \mathrm{M}_{\odot}$ and $M_{\mathrm{sec}}=0.2661 \pm 0.0095 \mathrm{M}_{\odot}, R_{\mathrm{pri}}=$ $0.762 \pm 0.022 \mathrm{R}_{\odot}$ and $R_{\mathrm{sec}}=0.748 \pm 0.023 \mathrm{R}_{\odot}$, and $T_{\mathrm{pri}}=$ $3260 \pm 73 \mathrm{~K}$ and $T_{\mathrm{sec}}=3213 \pm 73 \mathrm{~K}$. The posterior mass and radius distributions are shown in Fig. 7 (in orange), along with the results from only modelling the light curves and RVs (in grey). Modelling the system SED simultaneously with the light curves and RVs, and hence being able to incorporate the spectroscopic light ratio from the HIRES spectra, has two effects: (i) the radius uncertainties reduce by a factor of $\sim 3$; and (ii) the radius posteriors change from extended, slightly bimodal, distributions to well-defined unimodal posteriors. This improvement primarily results from breaking the degeneracy between the radius ratio and the cosine of the inclination and, to a lesser extent, the surface brightness ratios, ${ }^{4}$ which is significant when modelling only the light curves and RVs. We feel these improvements justify our approach to model the light

\footnotetext{
${ }^{4}$ This is smaller because there are two light-curve bands, albeit in closely separated bandpasses.
} 


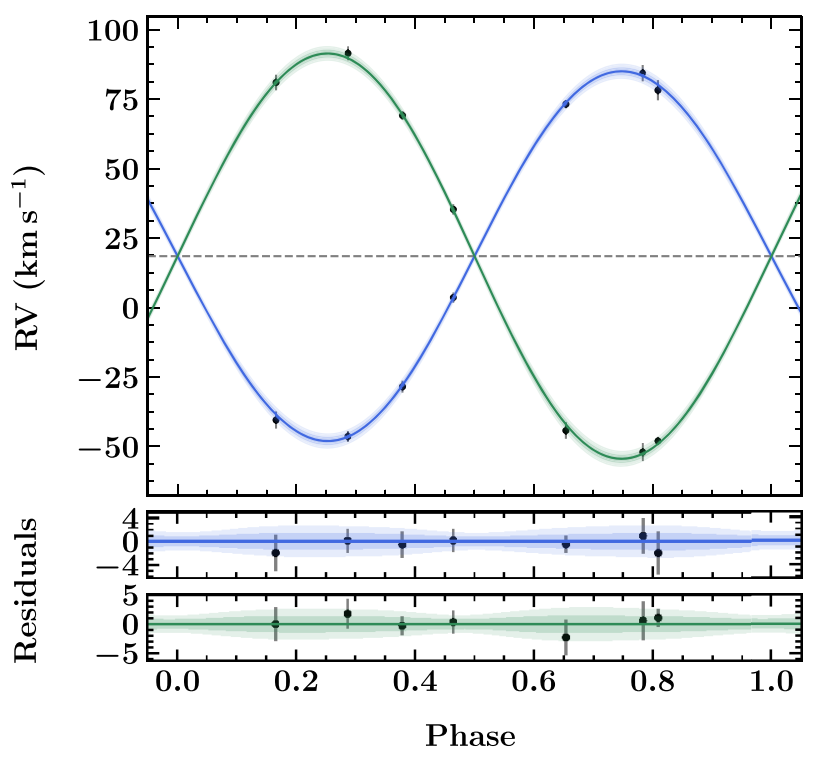

Figure 4. Phase-folded RV orbit of Mon-735. The lines and shaded regions indicate the median and $1 \sigma$ and $2 \sigma$ confidence intervals of the posterior RV orbits (blue and green for the primary and secondary stars, respectively), with Keck/HIRES RV measurements shown as black points. The grey horizontal dotted line indicates the systemic velocity. The two panels below show the residuals of the primary and secondary RV model orbits (top and bottom, respectively). The primary star's orbit has a slightly smaller semi-amplitude than the secondary star's orbit, suggesting a slightly higher primary star mass.

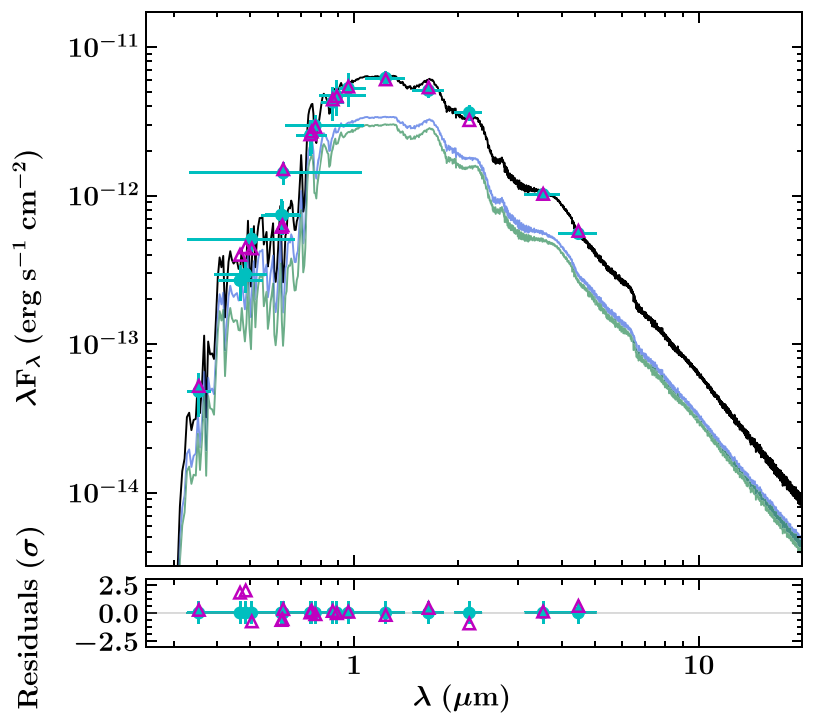

Figure 5. Spectral energy distribution (SED) of Mon-735. Cyan points represent the observed broad-band magnitudes reported in Table 2, which together comprise the observed SED. The horizontal cyan error bars indicate the spectral range of each band. SEDs constructed from BT-Settl model atmospheres for the primary and secondary stars are shown in blue and green, respectively. Their combined SED is shown in black with its prediction in each observed band indicated by magenta triangles. Residuals are shown below. The radii and effective temperatures of the two stars are similar, which results in comparable stellar SEDs.

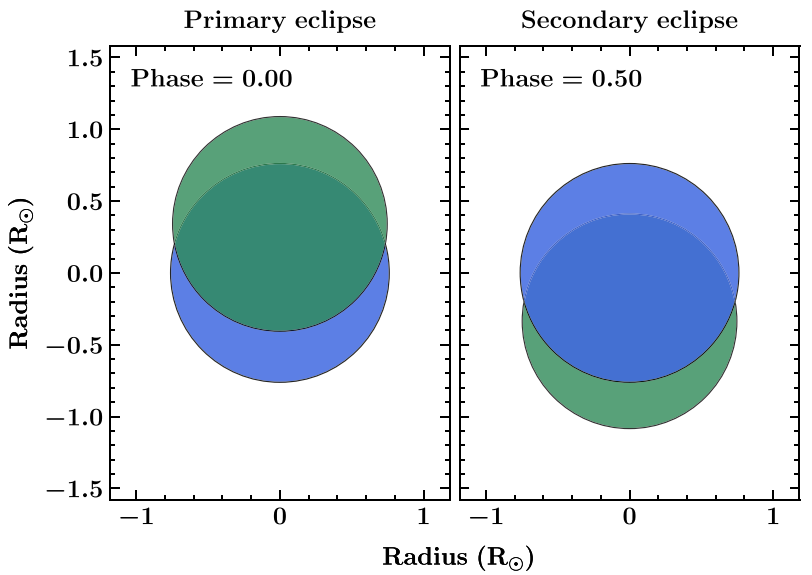

Figure 6. System geometry of Mon-735, to scale, as observed at primary and secondary eclipse (left and right, respectively). In both cases, the primary star is shown in blue and the secondary star in green. The fraction of each star occulted during eclipse is significant, which results in deep eclipses, yet they are still grazing due to the similar sizes of the two stars.

curves, RVs, and SED simultaneously. We note that the posterior masses are essentially unchanged, which is reassuring, as we would not expect the inclusion of the SED and spectroscopic light ratio to significantly affect these parameters. Finally, we tested models with constrained GP hyperparameters, such that the GP-EBOP variability model was essentially a flat line, and find consistent masses, radii, and effective temperatures.

\subsection{Consistent determination of fundamental parameters for CoRoT 223992193}

In addition to determining fundamental parameters for Mon-735, we also remodelled the other known low-mass EB in NGC 2264, CoRoT 223992193 (Gillen et al. 2014, hereafter G14), to determine updated fundamental parameters. Using GP-EBOP, we simultaneously modelled the CoRoT and Spitzer 3.6 and $4.5 \mu \mathrm{m}$ light curves, RVs from the Very Large Telescope (VLT)/Fibre Large Array Multi Element Spectrograph (FLAMES) and William Herschel Telescope (WHT)/Intermediate-dispersion Spectrograph and Imaging System (ISIS), and the system SED (see G14 and Gillen et al. 2017a for details of the data). This was done to obtain a consistent set of parameters that were determined using the same methodology as for Mon-735. These results will be reported in detail in a future publication. Here, we simply report the key parameters: $M_{\text {pri }}=0.665 \pm 0.015 \mathrm{M}_{\odot}$ and $M_{\mathrm{sec}}=0.486 \pm 0.012 \mathrm{M}_{\odot}$, $R_{\text {pri }}=1.2911_{-0.036}^{+0.031} \mathrm{R}_{\odot}$ and $R_{\mathrm{sec}}=1.118_{-0.039}^{+0.042} \mathrm{R}_{\odot}$, and $T_{\text {pri }}=$ $3680_{-52}^{+58} \mathrm{~K}$ and $T_{\mathrm{sec}}=3645_{-53}^{+58} \mathrm{~K}$. We note that these masses and radii are consistent with the values reported in G14 to within $1 \sigma$ (temperatures were not directly determined in G14). We further note that the masses and radii for both stars are consistent to within $1 \sigma$ both when we do and do not simultaneously model the SED, which gives us confidence that incorporating the SED into the fitting process does not bias our mass and radius measurements.

\section{DISCUSSION}

Determining fundamental stellar parameters (masses, radii, and effective temperatures) for stars in EBs provides an observational 
Table 4. Fitted and derived parameters for Mon-735.

\begin{tabular}{|c|c|c|c|}
\hline Parameter & Symbol & Value & Unit \\
\hline \multicolumn{4}{|c|}{ Fitted physical parameters } \\
\hline Orbital period & $P$ & $1.9751388 \pm 0.0000050$ & d \\
\hline Time of primary eclipse centre & $T_{\text {prim }}$ & $2456408.9725 \pm 0.0013$ & HJD \\
\hline Sum of radii & $\left(R_{\mathrm{pri}}+R_{\mathrm{sec}}\right) / a$ & $0.2767 \pm 0.0017$ & \\
\hline Radius ratio & $R_{\mathrm{sec}} / R_{\mathrm{pri}}$ & $0.981 \pm 0.054$ & \\
\hline Cosine of orbital inclination & $\cos i$ & $0.0621 \pm 0.0015$ & \\
\hline Eccentricity and argument of & $\sqrt{e} \cos \omega$ & $0.0006 \pm 0.0048$ & \\
\hline periastron combination terms & $\sqrt{e} \sin \omega$ & $-0.083_{-0.037}^{+0.072}$ & \\
\hline Systemic velocity & $V_{\text {sys }}$ & $18.47 \pm 0.60$ & $\mathrm{~km} \mathrm{~s}^{-1}$ \\
\hline Primary RV semi-amplitude & $K_{\text {pri }}$ & $66.5 \pm 1.1$ & $\mathrm{~km} \mathrm{~s}^{-1}$ \\
\hline Secondary RV semi-amplitude & $K_{\mathrm{sec}}$ & $72.9 \pm 1.1$ & $\mathrm{~km} \mathrm{~s}^{-1}$ \\
\hline Distance & $d$ & $718 \pm 27$ & $\mathrm{pc}$ \\
\hline Reddening & $A_{V}$ & $0.105_{-0.078}^{+0.150}$ & \\
\hline Primary effective temperature & $T_{\text {pri }}$ & $3260_{-63}^{+73}$ & K \\
\hline Secondary effective temperature & $T_{\mathrm{sec}}$ & $3213_{-64}^{+73}$ & $\mathrm{~K}$ \\
\hline \multicolumn{4}{|c|}{ Derived fundamental parameters } \\
\hline Primary mass & $M_{\text {pri }}$ & $0.2918 \pm 0.0099$ & $\mathrm{M}_{\odot}$ \\
\hline Secondary mass & $M_{\mathrm{sec}}$ & $0.2661_{-0.0089}^{+0.0095}$ & $\mathrm{M}_{\odot}$ \\
\hline Primary radius & $R_{\text {pri }}$ & $0.762 \pm 0.022$ & $\mathrm{R}_{\odot}$ \\
\hline Secondary radius & $R_{\mathrm{sec}}$ & $0.748 \pm 0.023$ & $\mathrm{R}_{\odot}$ \\
\hline Primary luminosity & $L_{\mathrm{pri}}$ & $0.0590_{-0.0052}^{+0.0064}$ & $\mathrm{~L}_{\odot}$ \\
\hline Secondary luminosity & $L_{\mathrm{sec}}$ & $0.0536_{-0.0048}^{+0.0056}$ & $\mathrm{~L}_{\odot}$ \\
\hline Primary surface gravity & $\log g_{\text {pri }}$ & $4.140 \pm 0.025$ & $\left(\mathrm{~cm} \mathrm{~s}^{-2}\right)$ \\
\hline Secondary surface gravity & $\log g_{\mathrm{sec}}$ & $4.116 \pm 0.026$ & $\left(\mathrm{~cm} \mathrm{~s}^{-2}\right)$ \\
\hline Mass sum & $M_{\mathrm{pri}}+M_{\mathrm{sec}}$ & $0.558 \pm 0.019$ & $\mathrm{M}_{\odot}$ \\
\hline Radius sum & $R_{\mathrm{pri}}+R_{\mathrm{sec}}$ & $1.508 \pm 0.019$ & $\mathrm{R}_{\odot}$ \\
\hline \multicolumn{4}{|c|}{ Derived radiative, orbital, and rotational parameters } \\
\hline Central surface brightness ratio in IRAC-1 & $J_{\text {IRAC-1 }}$ & $0.965 \pm 0.022$ & \\
\hline Central surface brightness ratio in IRAC-2 & $J_{\text {IRAC-2 }}$ & $0.971 \pm 0.018$ & \\
\hline Semimajor axis & $a$ & $5.453 \pm 0.060$ & $\mathrm{R}_{\odot}$ \\
\hline Orbital inclination & $i$ & $86.441_{-0.079}^{+0.088}$ & \\
\hline Eccentricity & $e$ & $0.0068_{-0.0062}^{+0.0075}$ & \\
\hline Longitude of periastron & $\omega$ & $-89_{-3}^{+13}$ & $\circ$ \\
\hline Primary synchronized velocity & $V_{\text {pri sync }}$ & $19.51 \pm 0.57$ & $\mathrm{~km} \mathrm{~s}^{-1}$ \\
\hline Secondary synchronized velocity & $V_{\text {sec sync }}$ & $19.15 \pm 0.59$ & $\mathrm{~km} \mathrm{~s}^{-1}$ \\
\hline
\end{tabular}

test of stellar evolution theory. Furthermore, for EBs in young open clusters, the distances determined from their modelling give independent estimates for their host cluster. We present an updated mass-radius relation for detached double-lined EBs in Section 5.1 and compare our results for Mon-735 and CoRoT 223992193 to the predictions of five stellar evolution models in Section 5.2. We then discuss the age and distance of NGC 2264 in Sections 5.3 and 5.4, before comparing the effect of using BT-Settl or PHOENIX model atmospheres in Section 5.5.

\subsection{Mass-radius relation for low-mass EBs}

Fig. 8 shows the mass-radius relation for detached double-lined EBs below $1.5 \mathrm{M}_{\odot}$. The coloured lines represent isochrones from Baraffe et al. (2015, hereafter BHAC15) from 1 Myr to 1 Gyr, and the data points show measurements for EBs in the field (grey) and in sub-Gyr open clusters (coloured, see figure caption for colour scheme). The two EBs in NGC 2264 are shown in blue, with the new system presented here, Mon-735, being the lower mass system and CoRoT 223992193 the higher mass system.

\subsection{Comparison with stellar evolution models}

We compare our mass, radius, and $T_{\text {eff }}$ measurements (and derived luminosity values) to the predictions of five sets of stellar evolution models in both the mass-radius and $T_{\text {eff }}-\log L$ planes. The massradius diagram (hereafter MRD) tests the fundamental (i.e. structural) predictions of the models, while the $T_{\text {eff }}-\log L$ diagram (i.e. Hertzsprung-Russell diagram, hereafter HRD) tests their predicted radiative properties. We use both Mon-735 and CoRoT 223992193 as a joint test of stellar evolution theory because they are both members of NGC 2264, and hence share the same composition and cluster age.

We compare to five sets of modern stellar evolution models: BHAC15 (Baraffe et al. 2015), MIST v1.2 (Choi et al. 2016; Dotter 2016), the standard and magnetic models of Feiden (2016, hereafter Feiden), and the PARSEC v1.1 models (Bressan et al. 2012). The BHAC15 models (Baraffe et al. 2015) are essentially an updated version of the BCAH98 models (Baraffe et al. 1998), which most notably now use BT-Settl model atmospheres and updated surface boundary conditions. The MIST v1.2 models (Choi et al. 2016; Dotter 2016) are based on the MESA (Modules for Experiments in Stellar Astrophysics) stellar evolution package. We use the rotating 


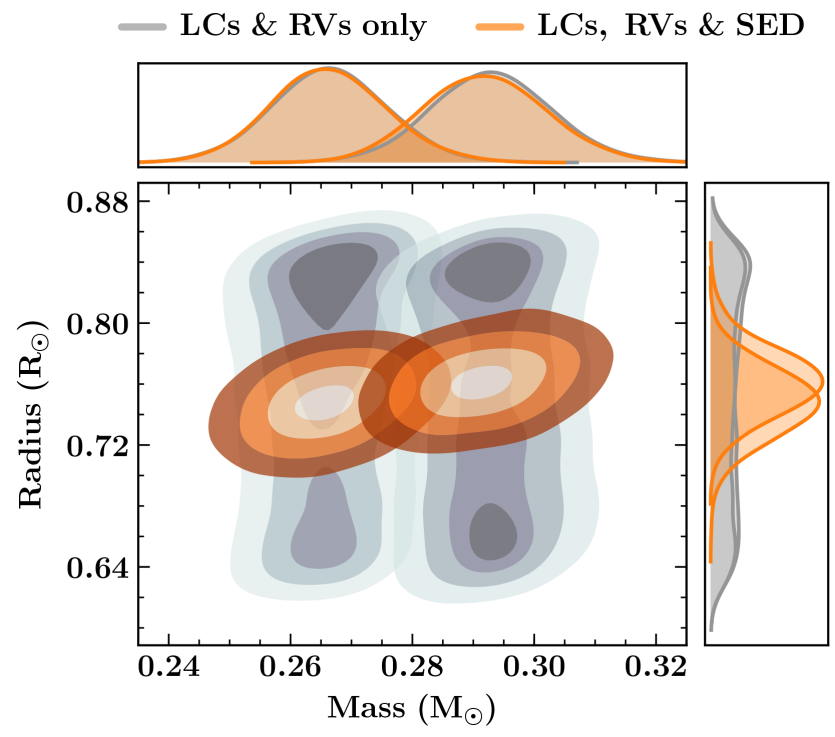

Figure 7. Assessing the effect of modelling the SED along with the light curves and RVs. Main plot: 2D posterior mass and radius distributions from modelling only the light curves and RVs (grey) compared to when the SED (and hence spectroscopic light ratio) is included in the fit (orange). The contours represent $0.5 \sigma, 1 \sigma, 1.5 \sigma$, and $2 \sigma$ boundaries. Top and right: 1D posterior distributions for the masses and radii, respectively. Including the SED in the modelling of Mon-735 significantly reduces the radius uncertainties and turns the slightly bimodal radius distributions into unimodal ones. There is no significant effect on the masses, as expected. set of isochrones but note that these are equivalent to the nonrotating versions on the PMS [solid-body rotation is commenced on the zero-age main sequence (ZAMS)]. The Feiden models are based on the Dartmouth Stellar Evolution Program (DSEP; Dotter et al. 2008), which were further developed in Feiden \& Chaboyer (2012, 2013) and Feiden (2016) to include the effect of magnetic fields. Magnetic fields act to inhibit convection and hence slow PMS contraction, which generally results in older age predictions compared to non-magnetic models. The PARSEC models (Bressan et al. 2012; Chen et al. 2014) are available in three versions: $\mathrm{v} 1.0$, v1.1, and v1.2S. Both the v1.0 and v1.1 models adopt the grey atmosphere approximation as their external boundary condition, which relates the temperature $(T)$ and the Rosseland mean optical depth $(\tau)$ across the atmosphere. The v1.2S models updated the $T-\tau$ relation to use the predictions of the BT-Settl models, but also included a shift in the $T-\tau$ relation to reproduce the observed mass-radius relation for low-mass dwarf stars. This shift means that the v1.2S models are not a direct test of the underlying stellar evolution theory for our two systems, and hence we compare to v1.1 models. We refer the reader to the relevant model papers for further details and to Stassun et al. (2014) for a general discussion of stellar evolution models in the context of PMS EBs.

In Figs 9-13, we compare our results to the BHAC15, MIST v1.2, Feiden (both standard and magnetic), and PARSEC v1.1 stellar evolution models, respectively. The top panels in these figures show mass-radius and $T_{\text {eff }}-\log L$ isochrones (coloured lines, left- and right-hand plots, respectively), along with the positions of Mon735 and CoRoT 223992193 (in green and blue, respectively). The

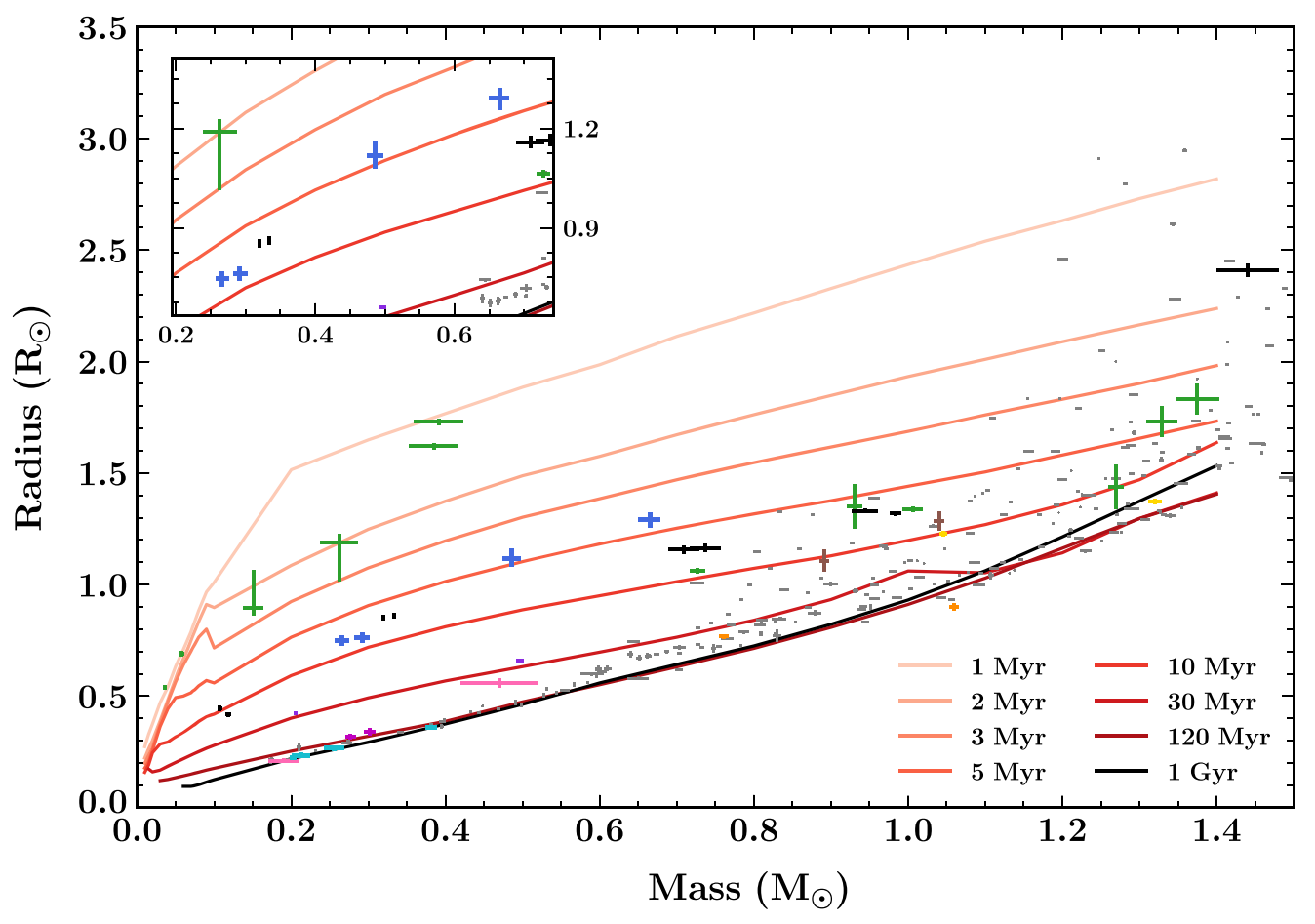

Figure 8. Mass-radius relation for detached double-lined EBs below $1.5 \mathrm{M}_{\odot}$. The coloured lines represent the solar metallicity isochrones of Baraffe et al. (2015, BHAC15) from 1 Myr to 1 Gyr (light-to-dark, top-to-bottom). Well-characterized EBs in sub-Gyr open clusters are coloured while field EBs are shown in grey. The two EBs in NGC 2264, Mon-735 and CoRoT 223992193, are plotted in blue, with other well-characterized cluster EBs shown in green (Orion), black (Upper Scorpius), pink (NGC 1647), gold (Per OB2), magenta (Pleiades), orange (Hyades), cyan (Praesepe), brown (Upper Centaurus Lupus), and purple (32 Orionis Moving Group). The cluster EBs are compiled from Gillen et al. (2017b, table 5), with subsequent additions from Gómez Maqueo Chew et al. (2019), David et al. (2019), and Murphy et al. (2020), and the field EBs from DEBCat (Southworth 2015). Inset: zoom on the region around Mon-735 and CoRoT 223992193. 


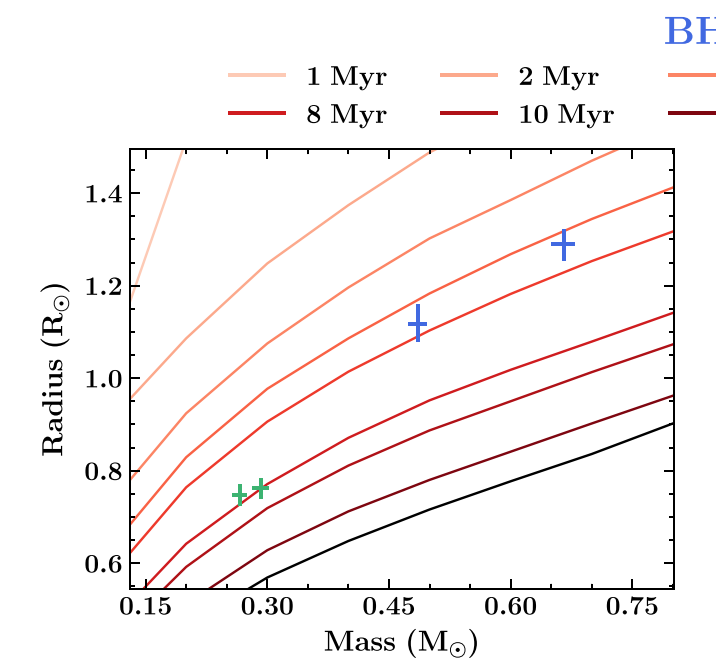

BHAC15
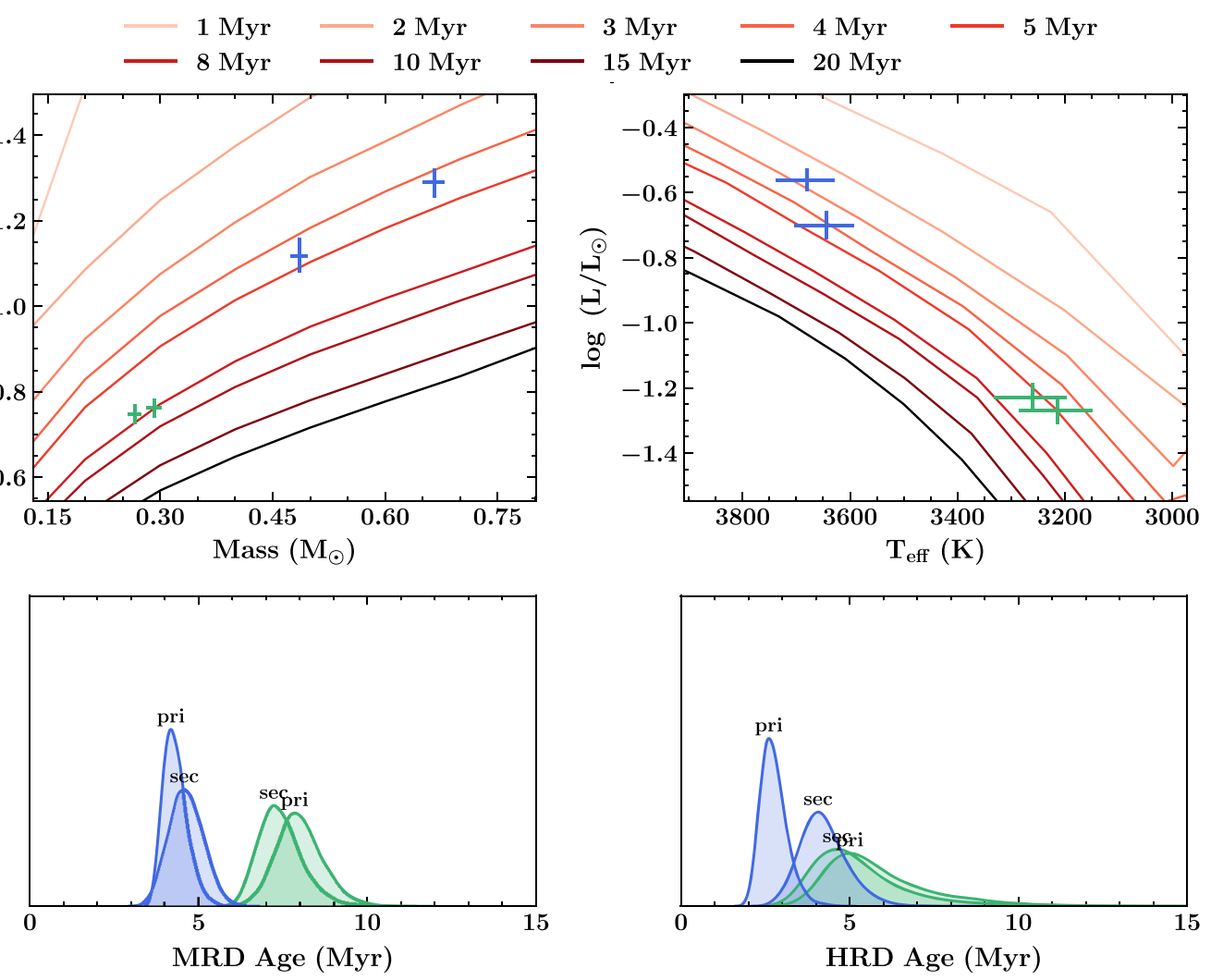

Figure 9. Comparison of the fundamental parameters of Mon-735 (green) and CoRoT 223992193 (blue) to the predictions of the BHAC15 stellar evolution models. Top row: mass-radius relation showing model isochrones between 1 and $20 \mathrm{Myr}$ (left), and the $T_{\text {eff }}-\log L$ relation with the same set of model isochrones. Bottom row: corresponding age distributions for Mon-735 (green) and CoRoT 223992193 (blue) based on their locations in the mass-radius diagram (MRD; left) and $T_{\text {eff }}-\log L$ diagram [i.e. Hertzsprung-Russell diagram (HRD); right]. The individual plots in Figs 9-13 all share the same axes for ease of comparison.

bottom panels show the model-predicted age distributions for each star based on their positions in the MRD and HRD (left- and right-hand plots, respectively). To estimate these ages, we first interpolated the models to compute a finer grid of predictions at each model age, and then compared our posterior distributions for Mon-735 and CoRoT 223992193 to this fine grid, which yielded a distribution of model-derived ages. These ages, along with the masses inferred from the positions of the stars in the HRD, are listed in Table 5.

From these plots, we can see five noteworthy trends: (i) Mon735 appears older than CoRoT 223992193 in the MRD for all models; (ii) Mon-735 appears slightly older than CoRoT 223992193 in the HRD for the non-magnetic models, but less so than in the MRD (and is comparable for the magnetic Feiden models); (iii) the age estimates for both components of each system in the MRD agree well, so while there is an apparent age difference between the two systems, the two stars within each system appear coeval, as we would expect; (iv) the coevality of the components within each system is more complicated in the HRD, where the stars in Mon-735 appear coeval but for CoRoT 223992193 the secondary appears older than the primary and in better agreement with Mon735; and (v) the model-derived age distributions are generally tighter from the MRD than the HRD (except for the primary star of CoRoT 223992193, where they are comparable), which results from the measured masses and radii being better constrained than the effective temperatures (and hence also luminosities).
Some subtler trends begin to emerge when we compare the results from different models. The left-hand panel of Fig. 14 shows the fractional difference between the MRD and HRD model ages. The primary and secondary components of Mon-735 are shown in blue and orange, and those of CoRoT 223992193 in pink and gold. The BHAC15, MIST v1.2, and Feiden (std) models (circles, squares, and upwards triangles) give very similar predictions for all stars. They find that both components of Mon-735, and the primary of CoRoT 223992193, are on average $\sim 30-35$ per cent $(\sim 2.5$ and 1.3 Myr, respectively) younger in the HRD than in the MRD. The HRD age of the CoRoT 223992193 secondary is slightly older than the primary and in agreement with its MRD age (slightly younger on average in the HRD but within or at the $1 \sigma$ level). This results from a higher estimated $T_{\text {eff }}$ ratio between the two components than the models would expect given their mass ratio. The PARSEC v1.1 models (diamonds) make predictions that are systematically younger than the other non-magnetic models in both the MRD ( 1.5 and 0.7 Myr for Mon-735 and CoRoT 223992193) and the HRD ( $\sim 2.4$ and 1.3 Myr). These result in a shift leftwards and upwards, respectively, which we see for all four stars in the plot. The magnetic Feiden models yield ages that are systematically older than the non-magnetic models in both the MRD and HRD. The MRD and HRD ages agree well for both components of Mon735 (comfortably within $1 \sigma$ ), the primary of CoRoT 223992193 appears $\sim 10$ per cent older in the HRD (but consistent at the $1 \sigma$ level), and the secondary appears $\sim 3 \mathrm{Myr}$ ( $\sim 50$ per cent) older in 


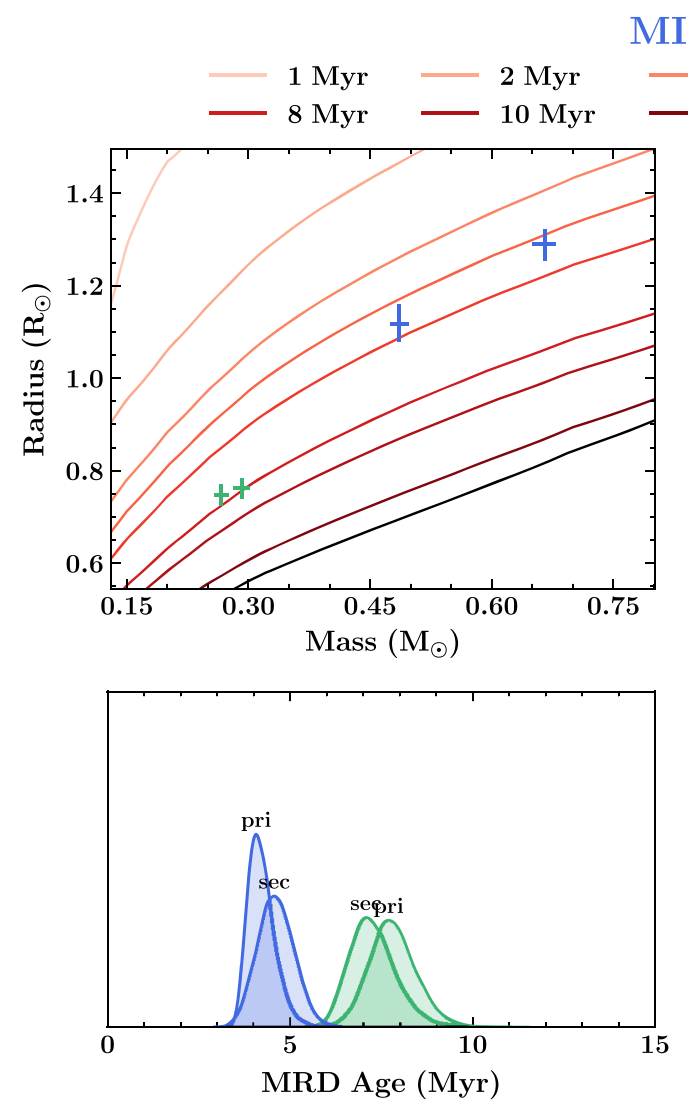

MIST v1.2
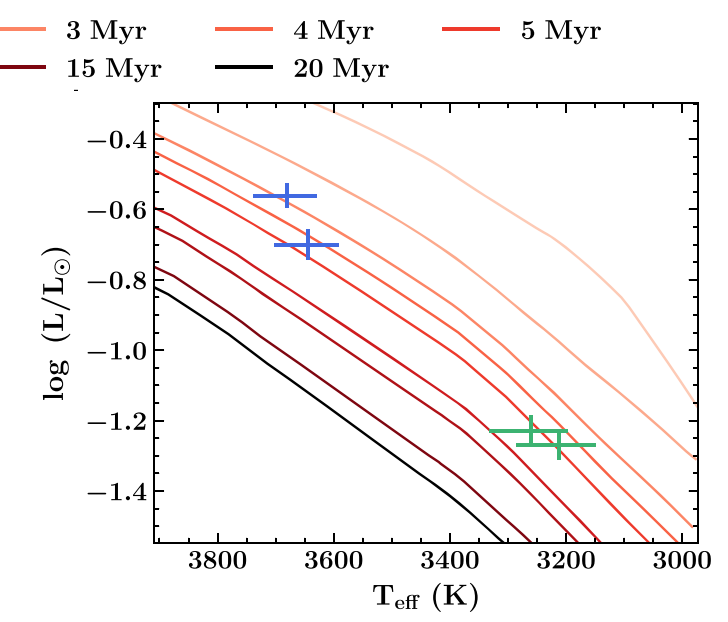

Figure 10. Same as Fig. 9, but comparing to MIST v1.2 models.

the HRD than the MRD. Interestingly, all models show the same overall trend: the two components of Mon-735 and the primary of CoRoT 223992193 have similar fractional age differences and the secondary of CoRoT 223992193 sits lower because it has a comparatively older age in the HRD compared to its MRD age. For the non-magnetic models, this brings its MRD and HRD ages into better agreement but into worse agreement for the magnetic Feiden models.

The right-hand panel of Fig. 14 shows the fractional differences between the dynamically determined masses (from our EB modelling) and the masses inferred from the positions of the stars in the HRD. This is a similar test to the fractional age differences shown in the left-hand panel, but reframed to investigate the masses we would infer for these stars given their positions in the HRD, and indeed we see similar trends. For Mon-735 and the primary of CoRoT 223992193, the masses inferred from the HRD positions of BHAC15, MIST v1.2, and Feiden (std) are on average smaller than the dynamical ones by $\sim 25-35$ per cent $(\sim 0.07$ and $0.2 \mathrm{M}_{\odot}$, respectively). The dynamical and HRD-inferred masses for the secondary of CoRoT 223992193 agree to within $1 \sigma$. The PARSEC v1.1 models give smaller HRD-inferred masses than the other non-magnetic models, which correspond to larger fractional mass differences of $\sim 55$ per cent $\left(\sim 0.15 \mathrm{M}_{\odot}\right)$ for Mon-735 and $\sim 30-45$ per cent for CoRoT $223992193\left(\sim 0.3\right.$ and $0.1 \mathrm{M}_{\odot}$ for the primary and secondary, respectively). The magnetic Feiden models predict larger HRD-inferred masses than the non-magnetic models. These are consistent with our dynamical masses for both components of Mon-735, $\sim 10$ per cent $\left(\sim 0.05 \mathrm{M}_{\odot}\right)$ larger for the primary of CoRoT 223992193 (but consistent to within 1 $\sigma$ ), and $\sim 40$ per cent $\left(\sim 0.2 \mathrm{M}_{\odot}\right)$ larger for the secondary of CoRoT 223992193.

For both components of Mon-735 and the primary of CoRoT 223992193, the magnetic Feiden models perform best with consistent MRD versus HRD ages and dynamical versus HRD masses. The properties of CoRoT 223992193's secondary are best matched by the non-magnetic models of BHAC15, MIST v1.2, and Feiden (std). Overall, there is no conclusive trend in the predicted ages and masses for all stars across models.

Finally, we note that both systems do not have known tertiary companions, which is important as Stassun et al. (2014) showed that EBs in triple systems are generally less-well explained by current stellar evolution theory than their counterparts without tertiary companions. The dynamical role of a tertiary companion in affecting the evolution of stars in binary configurations is an ongoing question, but one of significant interest (e.g. Gómez Maqueo Chew et al. 2012; Stassun et al. 2014; Cheng, Vinson \& Naoz 2019).

\subsection{The age of NGC 2264 from EBs}

NGC 2264 is typically considered to be $\sim 3_{-3}^{+5}$ Myr (Walker 1956; Park et al. 2000; Rebull et al. 2002). The two systems presented here appear to lie towards the upper end of this range, with Mon-735 and CoRoT 223992193 having apparent ages of 7-9 and $\sim 4-6 \mathrm{Myr}$, respectively (from their positions in the MRD). It is interesting to note that Upper Scorpius has typically been 

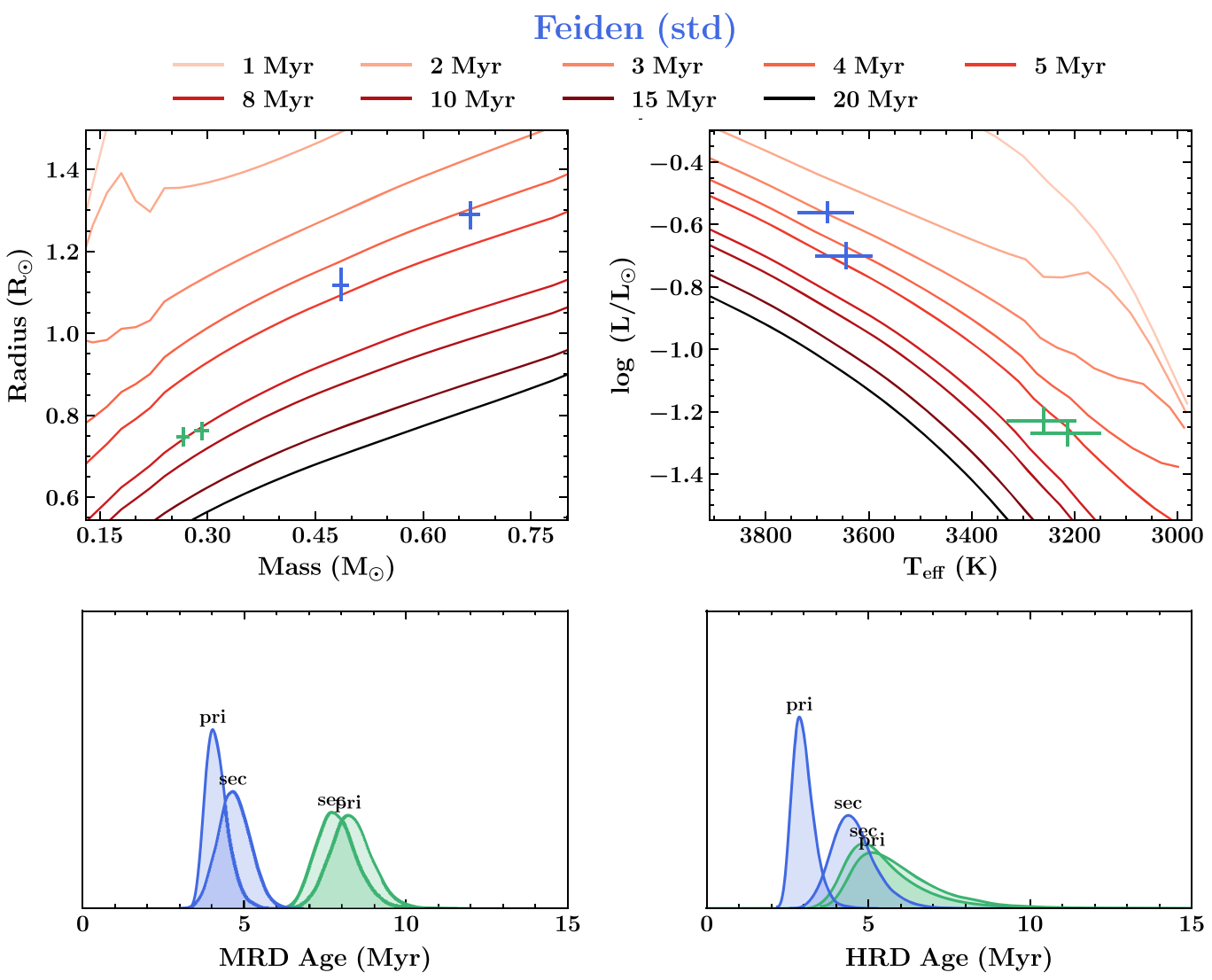

Figure 11. Same as Fig. 9, but comparing to the standard Feiden models.

considered older than NGC 2264 with an age of 5-10 Myr (e.g. Pecaut, Mamajek \& Bubar 2012; Feiden 2016; Pecaut \& Mamajek 2016; Rizzuto et al. 2016, and references therein). Recently, David et al. (2019) studied nine EBs in Upper Scorpius and found evidence for a self-consistent age of 5-7 Myr across the 0.3$5 \mathrm{M}_{\odot}$ mass range. Given that the ages of the EBs in NGC 2264 and Upper Scorpius are consistent, it is possible that the range of ages spanned by stars in these clusters is more similar than previously recognized. However, it should be kept in mind that the double-lined EBs that can be well measured via both high-precision photometry and precise spectroscopy in order to obtain their radii and masses, may be preferentially sampling the older, disc-free populations in young regions. Both NGC 2264 and Upper Scorpius are adjacent to on-going star formation within molecular cloud cores.

\subsection{The distance to NGC 2264 from EBs in the Gaia era}

The distance to NGC 2264 has generally been considered to be 700-800 pc (Sung et al. 1997; Dahm 2008; Sung \& Bessell 2010), although individual works have suggested a range between $\sim 400$ and 900 pc (e.g. Dzib et al. 2014 and Baxter et al. 2009, respectively). In G14, we determined a distance of $d=756 \pm 96 \mathrm{pc}$ to CoRoT 223992193. Subsequently, the Gaia DR2 parallax measurement yielded a consistent distance estimate of $d=745_{-68}^{+83}$ pc. From our new modelling, where we simultaneously solve for the distance to the system, we obtain $d=735_{-37}^{+51}$ pc. For Mon735 we determine a distance of $d=718 \pm 27 \mathrm{pc}$, which is in agreement with the Gaia DR2 estimate of $718_{-57}^{+67} \mathrm{pc}$. Both of our distance estimates are also consistent with Gaia DR2-derived distances to NGC 2264 of $738_{-21}^{+23}$ pc by Kuhn et al. (2019) and $719 \pm 16$ pc by Maíz Apellániz (2019). In our final modelling, we included the Gaia DR2 parallaxes as priors, but also tested models without them and find consistent distances $(d=715 \pm 29 \mathrm{pc}$ and $d=733_{-41}^{+57}$ pc for Mon-735 and CoRoT 223992193, respectively). This suggests that the distances inferred from our modelling are driven by the fit to the light curve, RV, and SED data.

\subsection{Differences between using BT-Settl and PHOENIX atmosphere models}

For the main results presented here, we opted to use BT-Settl model atmospheres in our SED modelling instead of PHOENIX v2 models. This was primarily because the BT-Settl models yielded distances that were in better agreement with Gaia, but also because they achieved a slightly better fit to the SED of Mon-735 (although the PHOENIX fit was still acceptable).

To test the effect of the stellar atmosphere model adopted, we performed additional modelling runs for both Mon-735 and CoRoT 223992193 with the PHOENIX models. Using the PHOENIX models for both Mon-735 and CoRoT 223992193 resulted in essentially unchanged masses and radii (easily consistent to within $1 \sigma$ ). The $T_{\text {eff }} \mathrm{s}$, however, were lower by $\sim 160 \mathrm{~K}$ for Mon-735 and $\sim 125 \mathrm{~K}$ for CoRoT 223992193. Correspondingly, the distances were smaller by $\sim 90$ and $\sim 80$ pc, respectively, which are both smaller than the Gaia distances but consistent to within $2 \sigma$. 


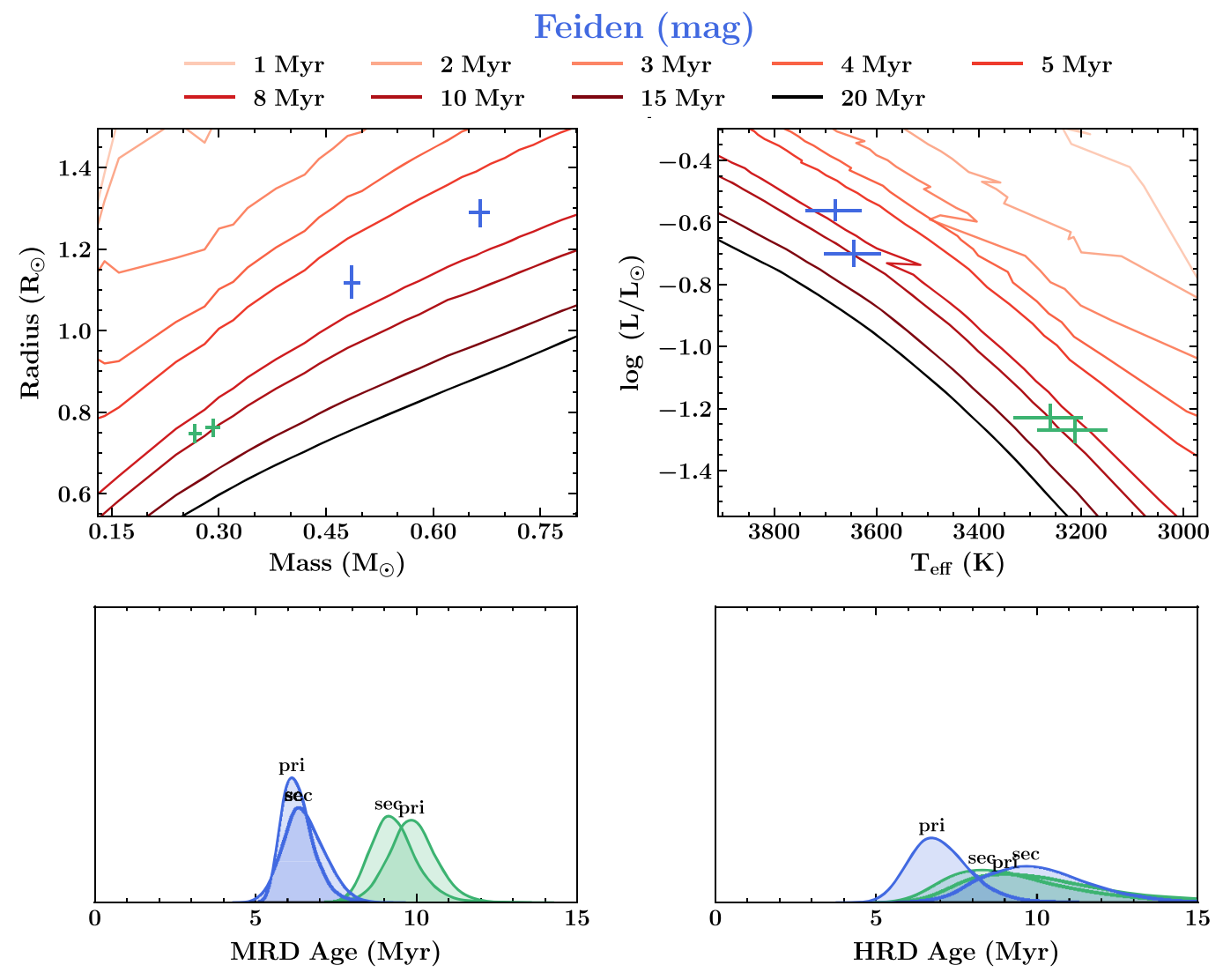

Figure 12. Same as Fig. 9, but comparing to the magnetic Feiden models.

With lower $T_{\text {eff }} \mathrm{s}$, the PHOENIX models suggest the stars are younger in the HRD by $\sim 1-3$ Myr for Mon-735 and $\sim 0.5-2 \mathrm{Myr}$ for CoRoT 223992193 (compared to the BT-Settl models). The positions in the MRD are essentially unchanged. In the fractional age and mass plots (Fig. 14), therefore, the values for each star shift upwards by $\sim 20-30$ per cent for Mon-735 and $\sim 10-30$ per cent for CoRoT 223992193, while the overall trend between stars remains similar. 5

\section{CONCLUSIONS}

We have presented the discovery and characterization of Mon-735 as a detached, double-lined PMS EB member of the NGC 2264 star-forming region. Mon-735 possesses IR Spitzer discovery light curves, as well as optical follow-up Keck/HIRES RVs. As the system displays relatively equal depth eclipses, modelling only the IR light curves and optical RVs suffered from a degeneracy between the radius ratio and inclination, and to a lesser extent the surface bright ratios, which led to large radius uncertainties. We overcame this issue by simultaneously modelling the system SED along with the light curves and RVs, which allowed us to apply a light ratio constraint from the HIRES spectra and propagate it through the SED model into the Spitzer light-curve bands and hence into the eclipse modelling; this broke the aforementioned degeneracy and

\footnotetext{
${ }^{5}$ The PARSEC v1.1 models do not extend down to the PHOENIX-derived $T_{\text {eff }}$ S for Mon-735 and hence were not included in this comparison.
}

yielded well-constrained radii. As a result of this effort, GP-EBOP now has the capability to simultaneously model light curves, RVs, and SEDs.

Simultaneously modelling the Spitzer light curves, Keck/HIRES RVs, and system SED, we determine self-consistent masses, radii, and temperatures for both component stars, as well as the distance to Mon-735 and, by extension, NGC 2264. We find that the two stars have masses of $M=0.2918 \pm 0.0099$ and $0.2661 \pm 0.0095 \mathrm{M}_{\odot}$, radii of $R=0.762 \pm 0.022$ and $0.748 \pm 0.023 \mathrm{R}_{\odot}$, and effective temperatures of $T_{\text {eff }}=3260 \pm 73$ and $3213 \pm 73 \mathrm{~K}$, and travel on circular orbits around their common centre of mass in $P=1.9751388 \pm 0.0000050 \mathrm{~d}$. In addition to Mon735, we also remodelled CoRoT 223992193, the other known low-mass EB in NGC 2264, to derive a consistent set of parameters for this system, so we could make a joint comparison for both systems to the predictions of different stellar evolution models.

We compare to the BHAC15, MIST v1.2, Feiden (both standard and magnetic), and PARSEC v1.1 models and find that the lower mass Mon-735 appears older than CoRoT 223992193 in the MRD and, to a lesser extent, in the HRD. In the MRD, Mon-735 appears to be 7-9 Myr, while CoRoT 223992193 appears to be 4-6 Myr. The two components in each EB system possess consistent MRD ages, whereas in the HRD, the components of Mon-735 are coeval but the secondary of CoRoT 223992193 appears slightly older than the primary, and in better agreement with Mon-735. For both components of Mon-735 and the primary of CoRoT 223992193, the magnetic Feiden models perform best with consistent MRD versus 


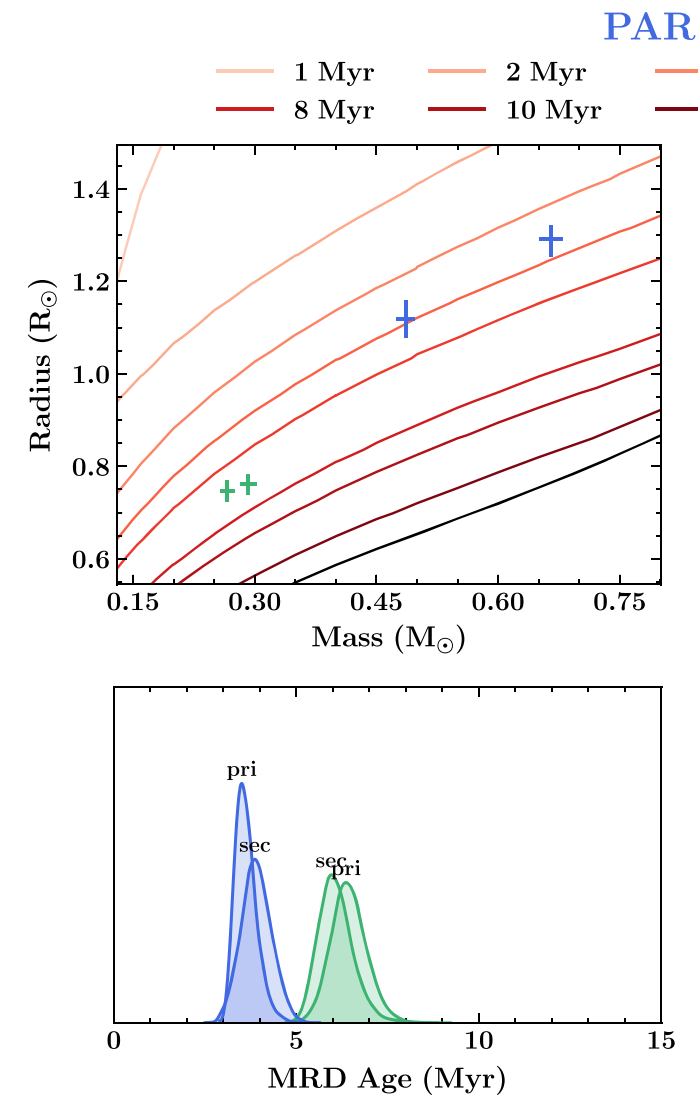

PARSEC v1.1
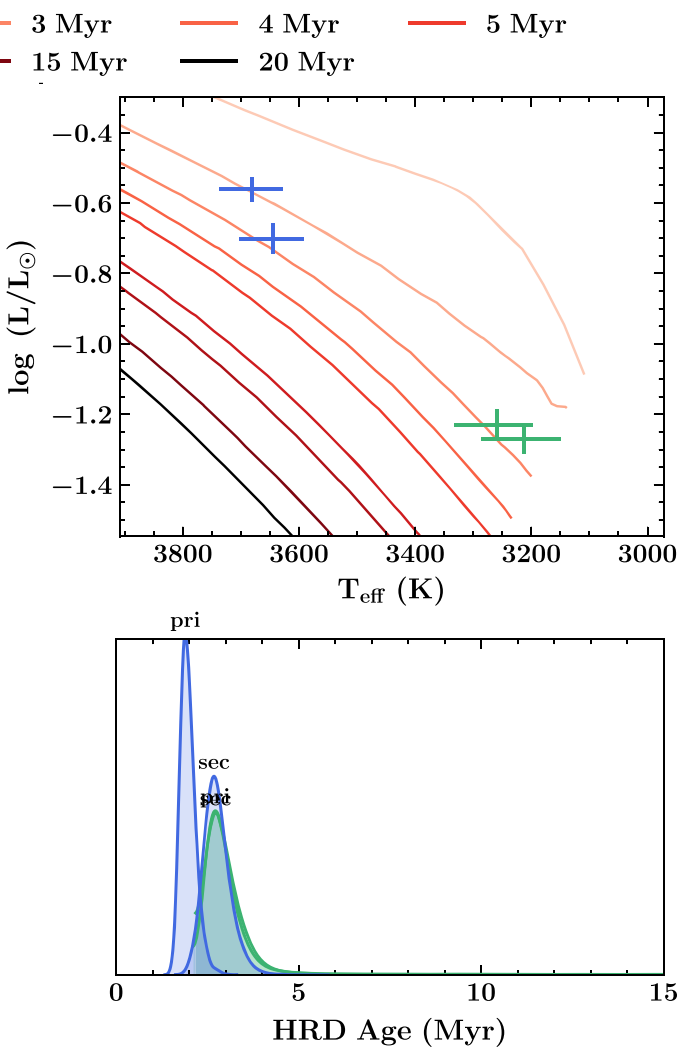

Figure 13. Same as Fig. 9, but comparing to PARSEC v1.1 models.

Table 5. Isochronal ages of Mon-735 and CoRoT 223992193 in the mass-radius diagram (MRD) and Hertzsprung-Russell diagram (HRD), and HRD-inferred masses.

\begin{tabular}{|c|c|c|c|c|c|c|}
\hline \multirow[t]{2}{*}{ Model } & \multicolumn{2}{|c|}{ MRD age (Myr) } & \multicolumn{2}{|c|}{ HRD age (Myr) } & \multicolumn{2}{|c|}{ HRD mass $\left(\mathrm{M}_{\odot}\right)$} \\
\hline & Primary & Secondary & Primary & Secondary & Primary & Secondary \\
\hline \multicolumn{7}{|c|}{ Mon-735 } \\
\hline BHAC15 & $8.0 \pm 0.7$ & $7.4 \pm 0.7$ & $5.5_{-1.0}^{+1.8}$ & $5.0_{-0.9}^{+1.5}$ & $0.22 \pm 0.05$ & $0.20 \pm 0.04$ \\
\hline MIST v1.2 & $7.8 \pm 0.7$ & $7.2 \pm 0.7$ & $5.5_{-1.2}^{+1.8}$ & $4.9_{-1.1}^{+1.7}$ & $0.22 \pm 0.04$ & $0.20 \pm 0.04$ \\
\hline Feiden (std) & $8.3 \pm 0.7$ & $7.8 \pm 0.7$ & $5.7_{-0.9}^{+1.5}$ & $5.2_{-0.7}^{+1.3}$ & $0.21 \pm 0.05$ & $0.18 \pm 0.04$ \\
\hline Feiden (mag) & $9.9 \pm 0.7$ & $9.3 \pm 0.8$ & $9.9_{-1.8}^{+2.7}$ & $9.0_{-1.6}^{+2.5}$ & $0.29 \pm 0.06$ & $0.26 \pm 0.05$ \\
\hline PARSEC v1.1 & $6.4 \pm 0.5$ & $6.0 \pm 0.5$ & $2.8 \pm 0.5$ & $2.8 \pm 0.5$ & $0.13 \pm 0.03$ & $0.12 \pm 0.02$ \\
\hline \multicolumn{7}{|c|}{ CoRoT 223992193} \\
\hline BHAC15 & $4.3 \pm 0.4$ & $4.6 \pm 0.5$ & $2.7 \pm 0.4$ & $4.2 \pm 0.8$ & $0.46 \pm 0.05$ & $0.46 \pm 0.04$ \\
\hline MIST v1.2 & $4.2 \pm 0.4$ & $4.6 \pm 0.5$ & $2.9 \pm 0.4$ & $4.4 \pm 0.8$ & $0.48 \pm 0.04$ & $0.47 \pm 0.05$ \\
\hline Feiden (std) & $4.1 \pm 0.4$ & $4.7 \pm 0.5$ & $3.0 \pm 0.4$ & $4.5 \pm 0.7$ & $0.47 \pm 0.04$ & $0.47 \pm 0.04$ \\
\hline Feiden (mag) & $6.3 \pm 0.5$ & $6.5 \pm 0.7$ & $6.9 \pm 1.0$ & $9.9 \pm 1.7$ & $0.72 \pm 0.06$ & $0.69 \pm 0.07$ \\
\hline PARSEC v1.1 & $3.6 \pm 0.3$ & $3.9 \pm 0.4$ & $1.9 \pm 0.2$ & $2.7 \pm 0.4$ & $0.37 \pm 0.03$ & $0.35 \pm 0.04$ \\
\hline
\end{tabular}

HRD ages and dynamical versus HRD masses. The MRD and HRD properties of CoRoT 223992193's secondary are best matched by the non-magnetic models of BHAC15, MIST v1.2, and Feiden (std). For these two low-mass PMS systems in NGC 2264, there is no clear trend between models in either the MRD- and HRD-derived ages or the HRD-inferred masses, except that the magnetic models predict older ages and higher masses than the non-magnetic models.
CoRoT 223992193 and Mon-735 are the first two low-mass EBs to come out of the CoRoT and Spitzer observations of NGC 2264, with more systems in preparation. These will form a powerful sample of near-coeval EB systems, formed from the same parent molecular cloud, with which to test PMS stellar evolution theory and better understand both the age of, and age spread within, the NGC 2264 region. 


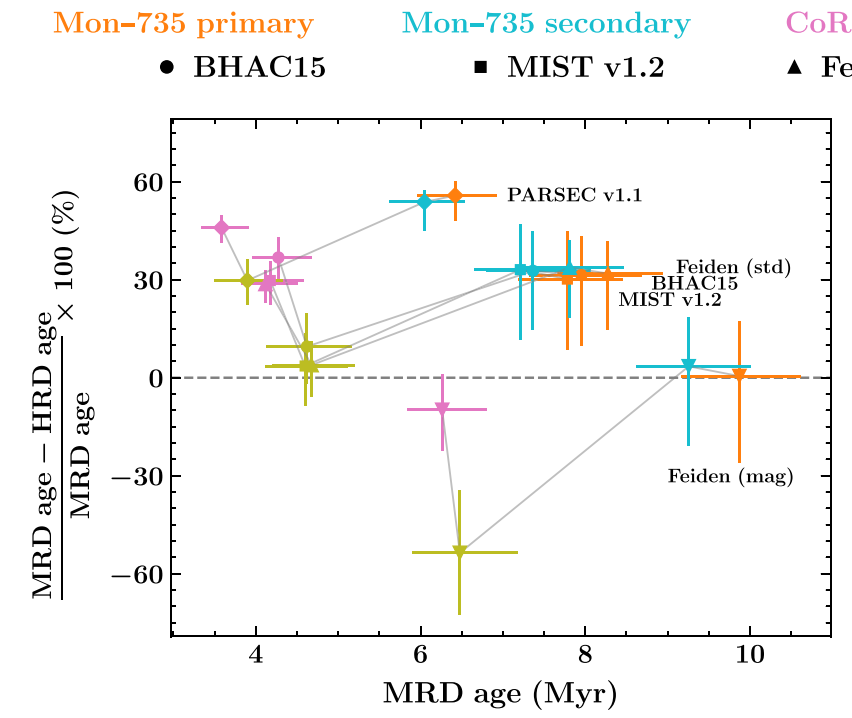

- PARSEC v1.1

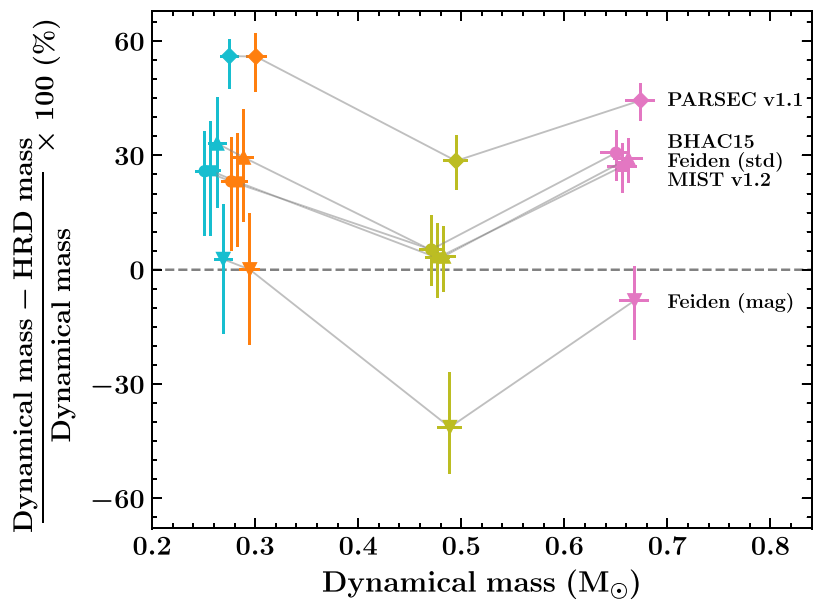

Figure 14. Left: fractional age difference between the MRD and HRD for Mon-735 and CoRoT 223992193 according to different PMS stellar evolution models. The colours represent different stars and the symbols represent different PMS models. Right: fractional mass difference between our dynamically determined masses and those predicted from the theoretical HRD according to different PMS models. On the $x$-axis, the different models for each star have been horizontally offset for clarity. The two plots test similar physics and hence show similar (mirrored) trends for the four stars.

\section{ACKNOWLEDGEMENTS}

The authors thank Hannu Parviainen, Trevor David, Grant Kennedy, and Didier Queloz for helpful discussions, and the anonymous referee for insightful reading of the manuscript and helpful suggestions for improvement. EG gratefully acknowledges support from the David and Claudia Harding Foundation in the form of a Winton Exoplanet Fellowship.

This work is based in part on observations made with the Spitzer Space Telescope, which is operated by the Jet Propulsion Laboratory, California Institute of Technology under a contract with NASA. Some of the data presented herein were obtained at the W. M. Keck Observatory, which is operated as a scientific partnership among the California Institute of Technology, the University of California, and the National Aeronautics and Space Administration. The Observatory was made possible by the generous financial support of the W. M. Keck Foundation. The authors wish to recognize and acknowledge the very significant cultural role and reverence that the summit of Maunakea has always had within the indigenous Hawaiian community. We are most fortunate to have the opportunity to conduct observations from this mountain. This research has made use of the VizieR catalogue access tool, CDS, Strasbourg, France (DOI: 10.26093/cds/vizier). The original description of the VizieR service was published in A\&AS, 143, 23 (Ochsenbein, Bauer \& Marcout 2000). This research has made use of the SIMBAD data base, operated at CDS, Strasbourg, France (Wenger et al. 2000). This research has made use of the SVO Filter Profile Service (FPS; http://svo2.cab.inta-csic.es/theory/fps/) supported from the Spanish MINECO through grant AYA201784089, and described in Rodrigo, Solano \& Bayo (2012). This publication makes use of data products from the Two Micron All Sky Survey, which is a joint project of the University of Massachusetts and the Infrared Processing and Analysis Center/California Institute of Technology, funded by the National Aeronautics and Space Administration and the National Science Foundation.

This work has made use of data from the European Space Agency (ESA) mission Gaia (https://www.cosmos.esa.int/gaia), processed by the Gaia Data Processing and Analysis Consortium (DPAC, https://www.cosmos.esa.int/web/gaia/dpac/consortium). Funding for the DPAC has been provided by national institutions, in particular the institutions participating in the Gaia Multilateral Agreement. Funding for the Sloan Digital Sky Survey IV has been provided by the Alfred P. Sloan Foundation, the U.S. Department of Energy Office of Science, and the Participating Institutions. SDSS-IV acknowledges support and resources from the Center for High-Performance Computing at the University of Utah. The SDSS web site is www.sdss.org. SDSS-IV is managed by the Astrophysical Research Consortium for the Participating Institutions of the SDSS Collaboration including the Brazilian Participation Group, the Carnegie Institution for Science, Carnegie Mellon University, the Chilean Participation Group, the French Participation Group, Harvard-Smithsonian Center for Astrophysics, Instituto de Astrofísica de Canarias, The Johns Hopkins University, Kavli Institute for the Physics and Mathematics of the Universe (IPMU) / University of Tokyo, the Korean Participation Group, Lawrence Berkeley National Laboratory, Leibniz Institut für Astrophysik Potsdam (AIP), Max-Planck-Institut für Astronomie (MPIA Heidelberg), Max-Planck-Institut für Astrophysik (MPA Garching), Max-Planck-Institut für Extraterrestrische Physik (MPE), National Astronomical Observatories of China, New Mexico State University, New York University, University of Notre Dame, Observatário Nacional / MCTI, The Ohio State University, Pennsylvania State University, Shanghai Astronomical Observatory, United Kingdom Participation Group, Universidad Nacional Autónoma de México, University of Arizona, University of Colorado Boulder, University of Oxford, University of Portsmouth, University of Utah, University of Virginia, University of Washington, University of Wisconsin, Vanderbilt University, and Yale University. The Pan-STARRS1 Surveys (PS1) and the PS1 public science archive have been made possible through contributions by the Institute for Astronomy, the University of Hawaii, the Pan-STARRS Project Office, the MaxPlanck Society and its participating institutes, the Max Planck Institute for Astronomy, Heidelberg and the Max Planck Institute for Extraterrestrial Physics, Garching, The Johns Hopkins University, 
Durham University, the University of Edinburgh, the Queen's University Belfast, the Harvard-Smithsonian Center for Astrophysics, the Las Cumbres Observatory Global Telescope Network Incorporated, the National Central University of Taiwan, the Space Telescope Science Institute, the National Aeronautics and Space Administration under Grant No. NNX08AR22G issued through the Planetary Science Division of the NASA Science Mission Directorate, the National Science Foundation Grant No. AST1238877, the University of Maryland, Eotvos Lorand University (ELTE), the Los Alamos National Laboratory, and the Gordon and Betty Moore Foundation.

\section{REFERENCES}

Affer L., Micela G., Favata F., Flaccomio E., Bouvier J., 2013, MNRAS, 430, 1433

Aguado D. S. et al., 2019, ApJS, 240, 23

Alencar S. H. P. et al., 2010, A\&A, 519, A88

Alexander R., 2008, New Astron. Rev., 52, 60

Allard F., Homeier D., Freytag B., 2012, Philos. Trans. R. Soc. Lond. Ser. A, 370, 2765

Andersen J., 1991, A\&AR, 3, 91

Baglin A., 2003, Adv. Space Res., 31, 345

Baraffe I., Chabrier G., Allard F., Hauschildt P. H., 1998, A\&A, 337, 403

Baraffe I., Homeier D., Allard F., Chabrier G., 2015, A\&A, 577, A42 (BHAC15)

Barentsen G., Vink J. S., Drew J. E., Sale S. E., 2013, MNRAS, 429, 1981

Barnes J. R., Collier Cameron A., Donati J.-F., James D. J., Marsden S. C., Petit P., 2005, MNRAS, 357, L1

Bastian N., Covey K. R., Meyer M. R., 2010, ARA\&A, 48, 339

Baxter E. J., Covey K. R., Muench A. A., Fưrész G., Rebull L., Szentgyorgyi A. H., 2009, AJ, 138, 963

Bell C. P. M., Mamajek E. E., Naylor T., 2015, MNRAS, 454, 593

Bouvier J. et al., 2007, A\&A, 463, 1017

Bouvier J. et al., 2016, A\&A, 590, A78

Bressan A., Marigo P., Girardi L., Salasnich B., Dal Cero C., Rubele S., Nanni A., 2012, MNRAS, 427, 127

Chambers K. C. et al., 2016, preprint (arXiv:1612.05560)

Chen Y., Girardi L., Bressan A., Marigo P., Barbieri M., Kong X., 2014, MNRAS, 444, 2525

Cheng S. J., Vinson A. M., Naoz S., 2019, MNRAS, 489, 2298

Choi J., Dotter A., Conroy C., Cantiello M., Paxton B., Johnson B. D., 2016, ApJ, 823, 102

Claret A., Hauschildt P. H., Witte S., 2012, A\&A, 546, A14

Cody A. M. et al., 2014, AJ, 147, 82

Cohen M., Wheaton W. A., Megeath S. T., 2003, AJ, 126, 1090

Czekala I., Mandel K. S., Andrews S. M., Dittmann J. A., Ghosh S. K., Montet B. T., Newton E. R., 2017, ApJ, 840, 49

Dahm S. E., 2008, in Reipurth B., ed., The Handbook of Star Forming Regions. Vol. I: The Northern Sky. Astron. Soc. Pac., San Francisco, p. 966

David T. J. et al., 2016a, AJ, 151, 112

David T. J. et al., 2016b, Nature, 534, 658

David T. J., Hillenbrand L. A., Gillen E., Cody A. M., Howell S. B., Isaacson H. T., Livingston J. H., 2019, ApJ, 872, 161

Dotter A., 2016, ApJS, 222, 8

Dotter A., Chaboyer B., Jevremović D., Kostov V., Baron E., Ferguson J. W., 2008, ApJS, 178, 89

Douglas S. T., Agüeros M. A., Covey K. R., Kraus A., 2017, ApJ, 842, 83

Dzib S. A., Loinard L., Rodríguez L. F., Galli P., 2014, ApJ, 788, 162

Evans D. W. et al., 2018, A\&A, 616, A4

Feiden G. A., 2016, A\&A, 593, A99 (Feiden)

Feiden G. A., Chaboyer B., 2012, ApJ, 761, 30

Feiden G. A., Chaboyer B., 2013, ApJ, 779, 183

Fitzpatrick E. L., 1999, PASP, 111, 63

Ford E. B., 2006, ApJ, 642, 505
Foreman-Mackey D., Hogg D. W., Lang D., Goodman J., 2013, PASP, 125, 306

Foreman-Mackey D., Agol E., Ambikasaran S., Angus R., 2017, AJ, 154, 220

Fưrész G. et al., 2006, ApJ, 648, 1090

Gaia Collaboration et al., 2018, A\&A, 616, A1

Gaidos E., Mann A. W., 2013, ApJ, 762, 41

Gillen E. et al., 2014, A\&A, 562, A50 (G14)

Gillen E. et al., 2017a, A\&A, 599, A27

Gillen E., Hillenbrand L. A., David T. J., Aigrain S., Rebull L., Stauffer J., Cody A. M., Queloz D., 2017b, ApJ, 849, 11 (G17)

Gillen E. et al., 2020, MNRAS, 492, 1008

Gómez Maqueo Chew Y., Stassun K. G., Prša A., Stempels E., Hebb L., Barnes R., Heller R., Mathieu R. D., 2012, ApJ, 745, 58

Gómez Maqueo Chew Y. et al., 2019, A\&A, 623, A23

Hartman J. D., Bakos G. Á., Kovács G., Noyes R. W., 2010, MNRAS, 408, 475

Herbig G. H., Soderblom D. R., 1980, ApJ, 242, 628

Husser T.-O., Wende-von Berg S., Dreizler S., Homeier D., Reiners A., Barman T., Hauschildt P. H., 2013, A\&A, 553, A6

Indebetouw R. et al., 2005, ApJ, 619, 931

Irwin J., Aigrain S., Hodgkin S., Irwin M., Bouvier J., Clarke C., Hebb L., Moraux E., 2006, MNRAS, 370, 954

Irwin J. et al., 2007, MNRAS, 380, 541

Irwin J. M. et al., 2011, ApJ, 742, 123

Irwin J. M. et al., 2018, AJ, 156, 140

Johnston C., Pavlovski K., Tkachenko A., 2019, A\&A, 628, A25

Jones J., White R. J., Quinn S., Ireland M., Boyajian T., Schaefer G., Baines E. K., 2016, ApJ, 822, L3

King J. R., Soderblom D. R., Fischer D., Jones B. F., 2000, ApJ, 533, 944

Kipping D. M., 2013, MNRAS, 435, 2152

Klagyivik P. et al., 2013, ApJ, 773, 54

Kraus A. L., Cody A. M., Covey K. R., Rizzuto A. C., Mann A. W., Ireland M. J., 2015, ApJ, 807, 3

Kraus A. L. et al., 2017, ApJ, 845, 72

Kuhn M. A., Hillenbrand L. A., Sills A., Feigelson E. D., Getman K. V., 2019, ApJ, 870, 32

Lamm M. H., Bailer-Jones C. A. L., Mundt R., Herbst W., Scholz A., 2004, A\&A, 417, 557

Livingston J. H. et al., 2018, AJ, 155, 115

López-Santiago J., Montes D., Gálvez-Ortiz M. C., Crespo-Chacón I., Martínez-Arnáiz R. M., Fernández-Figueroa M. J., de Castro E., Cornide M., 2010, A\&A, 514, A97

Maíz Apellániz J., 2019, A\&A, 630, A119

Mandel K., Agol E., 2002, ApJ, 580, L171

Mann A. W. et al., 2017, AJ, 153, 64

Mayne N. J., Naylor T., 2008, MNRAS, 386, 261

Meibom S., Mathieu R. D., Stassun K. G., 2009, ApJ, 695, 679

Meibom S., Barnes S. A., Platais I., Gilliland R. L., Latham D. W., Mathieu R. D., 2015, Nature, 517, 589

Morales-Calderón M. et al., 2012, ApJ, 753, 149

Murphy S. J. et al., 2020, MNRAS, 491, 4902

Ochsenbein F., Bauer P., Marcout J., 2000, A\&AS, 143, 23

Park B.-G., Sung H., Bessell M. S., Kang Y. H., 2000, AJ, 120, 894

Parviainen H., Aigrain S., 2015, MNRAS, 453, 3821

Pavlovski K., Southworth J., Tamajo E., 2018, MNRAS, 481, 3129

Pecaut M. J., Mamajek E. E., 2016, MNRAS, 461, 794

Pecaut M. J., Mamajek E. E., Bubar E. J., 2012, ApJ, 746, 154

Pepper J. et al., 2017, AJ, 153, 177

Perez M. R., The P. S., Westerlund B. E., 1987, PASP, 99, 1050

Rasmussen C. E., Williams C. K. I., 2006, Gaussian Processes for Machine Learning. the MIT Press, Cambridge, MA

Raynard L. et al., 2018, MNRAS, 481, 4960

Rebull L. M. et al., 2002, AJ, 123, 1528

Rebull L. M. et al., 2016a, AJ, 152, 113

Rebull L. M. et al., 2016b, AJ, 152, 114

Ribas Á., Bouy H., Merín B., 2015, A\&A, 576, A52

Rickman E. L. et al., 2019, A\&A, 625, A71 
Rizzuto A. C., Ireland M. J., Dupuy T. J., Kraus A. L., 2016, ApJ, 817, 164 Rodrigo C., Solano E., Bayo A., 2012, Technical Report, SVO Filter Profile Service Version 1.0

Schöfer P. et al., 2019, A\&A, 623, A44

Skrutskie M. F. et al., 2006, AJ, 131, 1163

Soderblom D. R., Hillenbrand L. A., Jeffries R. D., Mamajek E. E., Naylor T., 2014, in Beuther H., Klessen R. S., Dullemond C. P., Henning T., eds, Protostars and Planets VI. Univ. Arizona Press, Tucson, AZ, p. 219

Southworth J., 2015, in Rucinski S. M., Torres G., Zejda M., eds, ASP Conf. Ser. Vol. 496, Living Together: Planets, Host Stars and Binaries. Astron. Soc. Pac., San Francisco, p. 164

Stassun K. G., Mathieu R. D., Vaz L. P. R., Stroud N., Vrba F. J., 2004, ApJS, 151, 357

Stassun K. G., Feiden G. A., Torres G., 2014, New Astron. Rev., 60, 1

Stauffer J. R., Hartmann L. W., 1986, ApJS, 61, 531

Stauffer J. et al., 2014, AJ, 147, 83

Stauffer J. et al., 2015, AJ, 149, 130

Stauffer J. et al., 2016, AJ, 152, 115

Sung H., Bessell M. S., 2010, AJ, 140, 2070

Sung H., Bessell M. S., Lee S.-W., 1997, AJ, 114, 2644
Teixeira P. S. et al., 2006, ApJ, 636, L45

Tobin J. J., Hartmann L., Fûrész G., Hsu W.-H., Mateo M., 2015, AJ, 149, 119

Tonry J. L. et al., 2012, ApJ, 750, 99

Torres G., Andersen J., Giménez A., 2010, A\&AR, 18, 67

Triaud A. H. M. J. et al., 2011, A\&A, 531, A24

Venuti L. et al., 2014, A\&A, 570, A82

Vogt S. S. et al., 1994, in Crawford D. L., Craine E. R., eds, Proc. SPIE Vol. 2198, Instrumentation in Astronomy VIII. SPIE, Bellingham, p. 362

Walker M. F., 1956, ApJS, 2, 365

Wenger M. et al., 2000, A\&AS, 143, 9

Werner M. W. et al., 2004, ApJS, 154, 1

West A. A. et al., 2011, AJ, 141, 97

Williams J. P., Cieza L. A., 2011, ARA\&A, 49, 67

Young E. T. et al., 2006, ApJ, 642, 972

Zwintz K., Weiss W. W., 2006, A\&A, 457, 237

Zwintz K. et al., 2011, ApJ, 729, 20

This paper has been typeset from a $\mathrm{T}_{\mathrm{E}} \mathrm{X} / \mathrm{L} \mathrm{T} \mathrm{E} \mathrm{X}$ file prepared by the author. 